\title{
A Personal Information Management System
}

\author{
By \\ Qian Chen
}

\author{
A thesis submitted to \\ the Faculty of Graduate Studies and Research \\ in partial fulfillment of \\ the requirements for the degree of \\ Master of Computer Science
}

Ottawa-Carleton Institute for Computer Science

School of Computer Science

Carleton University

Ottawa, Ontario

January 16,2009

(C) Copyright

2009, Qian Chen 


$\begin{array}{ll}\begin{array}{l}\text { Library and } \\ \text { Archives Canada }\end{array} & \begin{array}{l}\text { Bibliothèque et } \\ \text { Archives Canada }\end{array} \\ \begin{array}{l}\text { Published Heritage } \\ \text { Branch }\end{array} & \begin{array}{l}\text { Direction du } \\ \text { Patrimoine de l'édition }\end{array} \\ \begin{array}{l}\text { 395 Wellington Street } \\ \text { Ottawa ON K1A 0N4 } \\ \text { Canada }\end{array} & \begin{array}{l}\text { 395, rue Wellington } \\ \text { Ottawa ON K1A 0N4 } \\ \text { Canada }\end{array}\end{array}$

Your file Votre référence ISBN: 978-0-494-47528-7

Our file Notre référence

ISBN: 978-0-494-47528-7

NOTICE:

The author has granted a nonexclusive license allowing Library and Archives Canada to reproduce, publish, archive, preserve, conserve, communicate to the public by telecommunication or on the Internet, loan, distribute and sell theses worldwide, for commercial or noncommercial purposes, in microform, paper, electronic and/or any other formats.

The author retains copyright ownership and moral rights in this thesis. Neither the thesis nor substantial extracts from it may be printed or otherwise reproduced without the author's permission.
AVIS:

L'auteur a accordé une licence non exclusive permettant à la Bibliothèque et Archives Canada de reproduire, publier, archiver, sauvegarder, conserver, transmettre au public par télécommunication ou par l'Internet, prêter, distribuer et vendre des thèses partout dans le monde, à des fins commerciales ou autres, sur support microforme, papier, électronique et/ou autres formats.

L'auteur conserve la propriété du droit d'auteur et des droits moraux qui protège cette thèse. $\mathrm{Ni}$ la thèse ni des extraits substantiels de celle-ci ne doivent être imprimés ou autrement reproduits sans son autorisation.
In compliance with the Canadian Privacy Act some supporting forms may have been removed from this thesis.

While these forms may be included in the document page count, their removal does not represent any loss of content from the thesis.
Conformément à la loi canadienne sur la protection de la vie privée, quelques formulaires secondaires ont été enlevés de cette thèse.

Bien que ces formulaires aient inclus dans la pagination, il n'y aura aucun contenu manquant.

\section{Canada}


The undersigned hereby recommend to

the Faculty of Graduate Studies and Research

acceptance of the thesis,

\title{
A Personal Information Management System
}

submitted by

\section{Qian Chen}

\author{
Dr. Douglas Howe \\ (Director, School of Computer Science)
}

Dr. Mengchi Liu

(Thesis Supervisor)

Carleton University

January, 2009 


\section{Abstract}

The increasing amount of heterogeneous personal information stored on personal computers has resulted in it being more difficult to attain efficient and effective Personal Information Management. Uniformly managing such information has become an increasingly important and complicated challenge.

In order to handle heterogeneous information, we propose a flexible data model called PIM Model, which unifies heterogeneous data and can represent various data models. Data information, structured information and relationship information among heterogeneous data sources can be represented as information items that have ids and sets of properties and values. Information items are connected through their ids to form a unified graphic view over all data sources.

Based on the PIM Model, we have implemented a Personal Information Management System, which uses Berkeley DB to store personal information, provides structured query and keyword search services, and enables users to customize properties, as well as classify and organize personal information. 


\section{Acknowledgements}

I would like to take this chance to sincerely thank my supervisor Dr. Mengchi Liu, a wonderful advisor, for his immense guidance, encouragement that helped me to carry on through difficult times. His inspiration, commitment and motivation for the research work have helped me to finish this thesis and I would like to thank him for his insights and comments as well. I would also like to give a special thanks to my parents for their immense support and love. I would like to thank and express my gratitude to all my colleagues and especially Mr. Ming Zhong for sharing his ideas during my research. I would also like to appreciate the help of Ms. Mansee Mongia for my thesis's proof reading. Last but not the least, I would like to thank the faculty and staff at the School of Computer Science for their support and encouragement and helped me achieve an exceptional education experience. 


\section{Contents}

Abstract iii

Acknowledgements $\quad$ iv

List of Tables $\quad$ ix

List of Figures $\quad$ xi

1 Introduction 1

1.1 Motivation ....................... 1

1.1.1 Representation of heterogeneous personal information . . . . . . 4

1.1.2 Querying personal information .............. 5

1.2 Objective ........................ 5

1.3 Outline of the Thesis . . . . . . . . . . . . 7

2 Related work $\quad 8$

2.1 Background ..................... 8

2.1 .1 A brief history .................. 8

2.1 .2 Related Terms . . . . . . . . . . . . . . 10

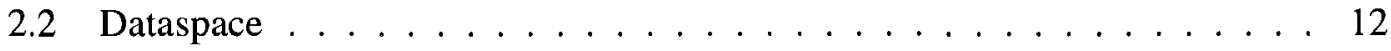

2.3 Data Model . . . . . . . . . . . . . . . . . . . . . 14 


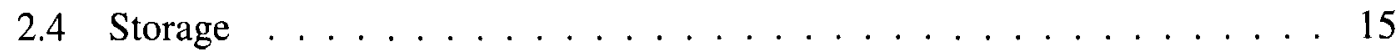

2.4 .1 Horizontal Schema . . . . . . . . . . . . . 15

2.4 .2 Vertical Schema ................... 17

2.4 .3 Approaches in RDF Store . . . . . . . . . . . . . . . . 19

2.4.3.1 RDF Triples Table . . . . . . . . . . . . 19

2.4.3.2 Property Tables ... . . . . . . . . . . 19

2.4.3.3 Vertically Partitioned Approach . . . . . . . . . . . . 21

2.4 .4 Interpreted Format $\ldots \ldots . \ldots . \ldots 21$

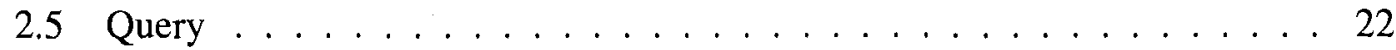

2.5.1 Triple query language $\ldots \ldots \ldots 23$

2.5 .2 Indexing approaches . . . . . . . . . . . . . 24

2.6 Current PIM system and our PIM system . . . . . . . . . . . . . 26

2.6.1 A brief survey of PIM systems . . . . . . . . . . . . 26 26

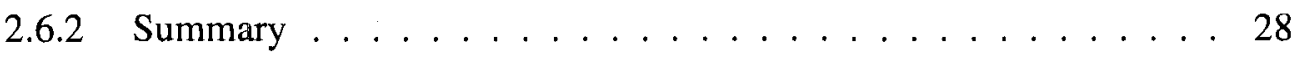

3 PIM Model $\quad 3$

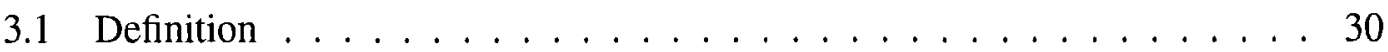

3.1 .1 Information Item $\ldots \ldots \ldots 31$

3.1 .2 Information Item Classes . . . . . . . . . . . . . . . . 32

3.1 .3 Class Hierarchy $\ldots \ldots . \ldots . \ldots . \ldots 33$

3.2 Representation of other models with PIM Model . . . . . . . . . . . . . 34

3.2.1 File System to PIM Model . . . . . . . . . . . . . . . . . 34

3.2.1.1 Representation of a folder . . . . . . . . . . 35

3.2.1.2 Representation of a file . . . . . . . . . 37

3.2.1.3 Representation of file system . . . . . . . . . . . 39

3.2.2 Relational Model to PIM Model . . . . . . . . . . . . . . . 41

3.2.2.1 Representation of a relational database . . . . . . . . 41 
3.2.2.2 Representation of a relation . . . . . . . . . . 42

3.2.2.3 Representation of a relational tuple . . . . . . . . 43

3.2 .3 XML Document to PIM Model . . . . . . . . . . . . . . . . . . . 44

3.2.3.1 Representation of a root node . . . . . . . . . . . 44

3.2.3.2 Representation of an XML element . . . . . . . . . 45

3.2.3.3 Representation of processing introduction . . . . . . . 46

3.2 .4 Emails to PIM Model . . . . . . . . . . . . . . . . . . . . . 48

3.3 Formation of a graph view . . . . . . . . . . . . . 51

3.4 Features of the PIM Model . . . . . . . . . . . . . . . . . . 54

4 Implementation $\quad 55$

4.1 System overview . . . . . . . . . . . . . . 55

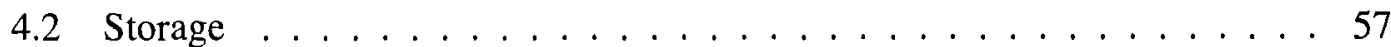

4.3 Data Collection ...................... 61

4.3 .1 Data Extraction . . . . . . . . . . . . . . 61

4.3 .2 Data Loading . . . . . . . . . . . . . . . . . . 63

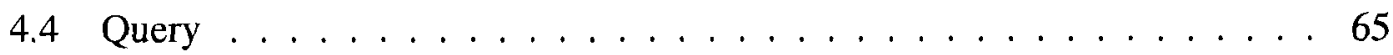

4.4 .1 Structured Query . . . . . . . . . . . . . . 66 66

4.4 .2 Structured Query Processing . . . . . . . . . . . . . . . 70

4.4.2.1 Execution Components . . . . . . . . . . . . 71

4.4.2.2 Class Execution in Query Join Graph Execution . . . . . 73

4.4 .3 Keyword Search . . . . . . . . . . . . . . 76

4.4.4 Keyword Search Processing . . . . . . . . . . . . . . 78

4.5 User interfaces $\ldots \ldots \ldots \ldots$. . . . . . . . . . . . . 80

4.6 Experimental results $\ldots \ldots \ldots \ldots$. . . . . . . . . 85

5 Conclusion $\quad 89$ 


\section{List of Tables}

2.1 Comparison of Dataspace and traditional data management architecture . . 13

2.2 Horizontal Schema . . . . . . . . . . . . . . . . 16

2.3 Vertical Schema . . . . . . . . . . . . . . . 18

2.4 Property Clustered Table . . . . . . . . . . . . . . . 20

2.5 Property Class Table . . . . . . . . . . . . . . . . . 21

2.6 Vertically Partitioned Approach _................. 22

2.7 Language Comparison . . . . . . . . . . . . . . . 25

2.8 Comparison of different PIM Systems . . . . . . . . . . . . . . . . . . 29

4.1 keyword inverted index . . . . . . . . . . . . . . . 78

4.2 Example of the Inverted Index $\ldots \ldots \ldots$

4.3 Extraction time and size of database as number of information items scales 86 


\section{List of Figures}

2.1 Example heterogeneous data set .................. 16

2.2 Store an Entity into Interpreted Format . . . . . . . . . . . . . . 23

3.1 Example classes structure . . . . . . . . . . . . . . . . 34

3.2 A folder's attribute information . . . . . . . . . . . . . . 36

3.3 A folder's relationship information . . . . . . . . . . . . . 36

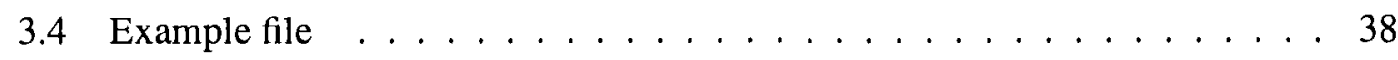

3.5 Example $x$ ml document . . . . . . . . . . . . . . . . . 38

3.6 Example file system data . . . . . . . . . . . . . . . . . 40

3.7 Example relational database $\ldots \ldots \ldots 42$

3.8 Example XML elements . . . . . . . . . . . . . . . . 46

3.9 Example processing instruction nodes . . . . . . . . . . . . . 47

3.10 Example email message . . . . . . . . . . . . . . . . . 50

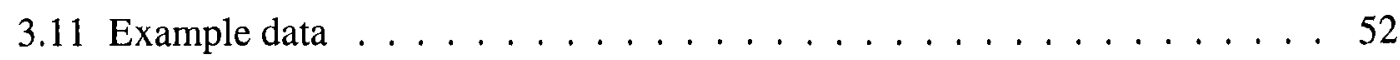

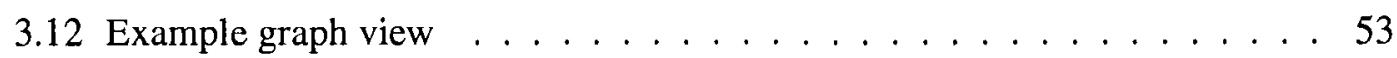

4.1 System Architecture . . . . . . . . . . . . . . . . . . . . 58

4.2 The schema of the databases $\ldots \ldots \ldots \ldots \ldots$

4.3 Store data in databases $\ldots \ldots \ldots \ldots 62$

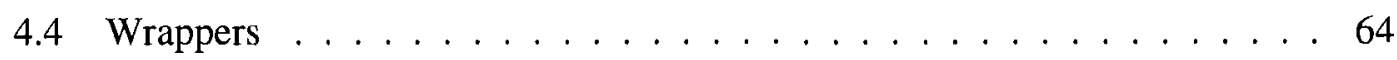

4.5 Algorithm of inserting a property . . . . . . . . . . 65 
4.6 Algorithm of inserting an information item . . . . . . . . . . . 66

4.7 Algorithm of inserting a property/object pair . . . . . . . . . . . . 67

4.8 Query Processing . . . . . . . . . . . . . . . . 70

4.9 Query Execution ........................ 71

4.10 Query Join Graph Execution . . . . . . . . . . . . . . . . 72

4.11 Classes Information . . . . . . . . . . . . . . . . . . 74

4.12 Algorithm of getting descent classes . . . . . . . . . . . . . . 76

4.13 Algorithm of getting ancestor classes . . . . . . . . . . . . . 77

4.14 Keyword Search Processing . . . . . . . . . . . . . . . 81

4.15 Keyword Search Processing Example . . . . . . . . . . . . . . . 81

4.16 Browse console . . . . . . . . . . . . . . . . . . . . 82

4.17 Classification console . . . . . . . . . . . . . . . 83

4.18 Keyword search console . . . . . . . . . . . . . . . . . . . . 84

4.19 Property search console . . . . . . . . . . . . . . . . . . . 85

4.20 Structured query console . . . . . . . . . . . . . . 86

4.21 Extraction time and size of database as number of information items scales 87

4.22 Query time as number of information items scales . . . . . . . . . . . . 88 


\section{Chapter 1}

\section{Introduction}

\subsection{Motivation}

People store various information on their personal computers for later usage. As the amount of information stored increases, so does the difficulty in locating it. Personal Information Management (PIM) is a term referring to both the practice and the study of the activities a person performs in order to acquire or create, store, organize, maintain, retrieve, use and distribute the information needed to meet life's many goals (everyday and long-term, work-related and not) and to fulfill life's many roles and responsibilities (as parent, spouse, friend, employee, member of community, etc.) [50]. PIM places special emphasis on the organization and maintenance of personal information collections in which information items, such as paper documents, electronic documents, email messages, web references, handwritten notes, etc., are stored for later use and repeated re-use. PIM systems have been proposed to save time and effort, and improve efficiency in locating and organizing personal information [31].

Commercial PIM systems, such as Google Desktop [13], Window Desktop Search [16] and searching functionalities within operating systems, are developed to find the information on personal computers. They mainly support the following types of items: Outlook 
email, Outlook Express, Word, Excel, PowerPoint, Internet Explorer, Pdf, Images, Music files, Videos, Zip files, Text files and web histories. Such systems index file names, directory names, meta data and content of supported documents and provide the full text search functionality. The main advantage of such systems is that they return search results fast. The main disadvantages are as follows:

- A large number of indexing consumes a lot of disk and memory space.

- There is no access to some special applications. For example, we store teaching information in a relational database table, in which course names, teacher information and room numbers are all stored. Through commercial PIM systems, we cannot find data in this database table.

- Commercial PIM systems only simply support keyword search. There is no way to search structured information and semi-structured information stored on personal computers. Because commercial PIM systems only rely on indexing technologies so that they consider all files as unstructured data and discard structured information and unstructured information among files. If users want to find files which contain one or several specific keywords in their meta data, contents and directory names, commercial PIM systems are good choices; however, if users want to find structured information among files, they are the worst assistants. For example, we want to search all children elements of an element in an Extensible Markup Language (XML) document. We input the name of the element into a desktop search tool and it returns a list of files which contains the keyword. The returning result is far from meeting the request - a list of names of the children elements.

Commercial PIM systems are not enough to search all personal information for the following reasons: personal information is stored in various applications; we may need different methods to manipulate data stored in those applications. Personal information 
is stored and presented in various formats by different applications. We may have structured data stored in relational databases, semi-structured data stored in XML documents, semi-structured data retained in email clients such as Outlook, structured, semi-structured and unstructured data residing at the file system and many other applications. Different search methods are needed in different applications. To access a relational database and to query data in it, we need Structure Query Language (SQL) and a JDBC connection. To retrieve an XML document's information including its data information and the structured information, we may use XQuery. To get information in emails, we may need to use email clients. To acquire the structured information in the file system, we may check folders and files manually. To query data stored in other specific applications, we may use specific programs or methods.

Though we can search information in each application, there is no way to search uniformly and we may need several steps in the searching behaviour. Imagine the case where we do not know in which application the information stores, we may need to start each application and search the information. For example, we store course schedule information in a relational table. Suppose that when we need to check the course schedule, we have already forgotten where it is. We may open desktop search to search if it exists in files and Outlook; we may open database management systems to search if it is in database tables; and we might open other applications to search if they are in those applications. In the worst case, we will need to open many various applications on the computer so as to get the information we need, which is a waste of time and inconvenient.

The reasons why we are not able to search personal information uniformly are as follows: personal information is heterogeneous; there is not an effective and efficient way to manage such information. The inconvenience caused by the heterogeneity of information also exists in the field of web search. The heterogeneity of web pages increases the difficulty of searching information contained in such pages. Nowadays, web pages have too many different formats: pages in HTML formats; pages in non-HTML formats 
like Pdf, Word, Excel, Powerpoint; script-based pages; pages generated dynamically by some database softwares and so on. Current search engines cannot find all kinds of pages. Web pages which cannot be found by search engines are called deep web, which includes: pages in non-HTML formats; script-based pages and pages generated dynamically by some database softwares.

After analyzing problems in PIM systems and issues of heterogeneous information, we find that there is an urgent requirement to effectively and efficiently manage heterogeneous data.

\subsubsection{Representation of heterogeneous personal information}

Dataspace [7] is a new data management architecture. It organizes all structured, semistructured and unstructured data in a uniform flexible schema-free model. The data dominated by Dataspace is loosely connected by its relationships to form a unified graphic view. Then, the Dataspace system is able to provide basic querying functionality such as keyword searching and graph-pattern querying on the view. Further, additional efforts can be applied to integrate data more tightly, such as conducting semantic integration, in order to provide more advanced services when users ask for. Consequently, systems under Dataspace can effectively and efficiently manage heterogeneous data and provide services for users sooner.

As a result, the main research challenge of the thesis is to determine how to deal with heterogeneous personal information using the vision Dataspace. The most important step for managing heterogeneous data in this vision is to find a proper model to properly and uniformly represent heterogeneous data sources. Once they are represented in a uniform view, then it is possible to operate on the view. 


\subsubsection{Querying personal information}

Once we have a unified view of a user's personal information, the next natural challenge is to determine how to query that view. In order to fully discuss user queries, we have to analyze structured queries and keyword searches. Structured queries always return accurate query results, but it is not easy to use. Keyword searches are simple to use, but users cannot receive accurate search results. Consequently, we have determined that another research challenge entailed is how to reduce the complexity of the structured query, as well as how we could enhance the accuracy of the keyword search.

\subsection{Objective}

The overall goal of our work presented in this thesis is to propose a flexible data model, which unifies a variety of structured, semi-structured and unstructured data. The model should be able to represent data in various data models. Furthermore, our work intends to implement a Personal Information Management System which stores heterogeneous personal information, provides structured query and keyword search services, and enables users to customize properties, as well as classify and organize personal information.

The contribution of the thesis is that it presents a novel data model, PIM Model, and designs and implements a personal information management system. The features of the PIM Model are as follows:

- Data in heterogeneous data sources have different formats and diverse data types and it is difficult to represent them in a single table. Our PIM Model makes representation of such data easy by reducing the effort of predefining the schema. Heterogeneous data is converted to information items. Through their ids, information items can be connected to form a uniform view. 
- Information items can belong to multiple classes and classes can be organized hierarchically.

- Dataspace requires representation of heterogeneous data in a uniform way, requires data to be integrated loosely, requires to provide services upon coexistent data and allows coexistent data to be integrated further. Our PIM Model represents heterogeneous data in a uniform graph view. Over the view, structured query and keyword search services are able to be provided. The view has simple format and it allows semantic integration upon it. Therefore, it fulfills the Dataspace vision.

The improved features of our Personal Information Management systems, based on the PIM Model, are as follows:

- Our system provides a flexible way for representing personal information. All information interested by users is represented using information items that have ids and sets of properties and values. No matter what kind the original data is, structured, semi-structured or unstructured, all data information and relationship information among information items is all extracted and transferred into the PIM Model and stored into the storage.

- All structured information is reserved. File systems contain structured information that is presented by a folder and file hierarchy. For example, information outside a document, such as the structured information "Folder study contains folder papers", is extracted as part of information item that has instance id with a property and a value, among which the id is the folder study's id, the property is hasFolder and value is the id of folder papers. The inside information of a file contains its own structure information (XML documents generally present structured information by tags and elements), which is also extracted into information items. 
- Our system is portable and extendable. The rules on how to extract information into the PIM Model are all clearly predefined. The process of dividing information into instances of ids with sets of properties and values is developed as decomposer components. The components are plug-ins of the PIM Model and are all connected to the storage through a unified interface. When new applications are required in the future, more decornposers can be developed and plugged into the whole system. In this way, the system is flexible and can continue to be developed as required.

- We provide users with structured query and keyword search services. The structured query is similar to SQL and returns accurate results. Differing from the traditional keyword search, which only indexes text, the keyword search in our system is a combination of structured query and the traditional keyword search.

\subsection{Outline of the Thesis}

The rest of the thesis has four chapters. Chapter 2 provides the background knowledge that is relevant to comprehending this thesis, a brief discussion of the related work for our research, including Dataspace, models, storage strategies for storing heterogeneous data set and spares data set, triple query languages, indexing technologies and a survey of current PIM systems. Chapter 3 introduces the PIM Model, which is a flexible data model that uniforms heterogeneous data sources. We additionally present an explanation of how to transform data sources from varying models to the PIM Model, how to unify data into a view and we also present features of the PIM Model. Chapter 4 presents the implementation of our Personal Information Management System, which includes the storage, data extraction and loading algorithms and the structured query and keyword search processing. The chapter also includes the user interfaces and concludes with experimental examples. Chapter 5 concludes the thesis, lists our contributions, and describes recommended direction for future research. 


\section{Chapter 2}

\section{Related work}

The main goal of the thesis is to build a PIM system with heterogeneous personal information in Dataspace vision. To achieve the goal, Dataspace, data model, storage, query are four main parts which we concern during the development. We structure this chapter as: background, Dataspace, data model, storage, query and current PIM Systems.

\subsection{Background}

\subsubsection{A brief history}

PIM-related matters can be traced back to ancient times. Before the written word was invented, human memory was the primary means for information preservation. Studies related to PIM are evidenced in mnemonic techniques which were essentially information management applied to human memory.

After the written word appeared, information was increasingly rendered in documents, which was titled as paper-based information. To support paper-based information, tools, such as vertical filling cabinet, aiming at managing documents were invented.

The beginning of the modern dialog on PIM is the article "As we may think" [32], 
which was published at the close of World War II. The author of the article, Vannevar Bush recognized a problem with the sheer quantity of information being produced and its compartmentalization by an increasing specialization of scientific discipline: "The investigator is staggered by the findings and conclusions of thousands of other workers - conclusions which he cannot find time to grasp, much less to remember, as they appear". He expressed a hope that is "technology might be used to extend our collective ability to handle information and to break down barriers impeding the productive exchange of information". Furthermore, Bush described a "memex" as a device in which an individual stores all his books, records, and communications, and which is mechanized so that it may be consulted with exceeding speed and flexibility. The "memex" used small head-mounted cameras to record experiences and microfilm to store these experiences.

From the 1950 s to 1980 s, increasing availability of computers made researcher to relate computers and personal information together. In 1950s, a computer was once considered as a source of metaphors and a test bed for efforts to understand human behaviour and thoughts. It showed that the computer could think like people do. After the positive result of the research in $1950 \mathrm{~s}$, the interests in the use of computers to help people think better and to process information more effectively increased.

The phrase "Personal Information Management" was first introduced in 1980's (Landsdale, 1988). PIM researchers expressed their excitement over the potential of the personal computer to greatly enhance the human ability to process and manage information. Moreover, the 1980s also saw the advent of so-called PIM tools which provided limited support for management of such things as appointments and scheduling, to-do lists, phone numbers and addresses. 


\subsubsection{Related Terms}

Important terms in PIM have been summarized on PIM Workshop, which was sponsored by the National Science Foundation of USA, held in January $27-29,2005$, Seattle. The most important terms in this area are presented as follows.

Information The original meaning of information is "information is a collaborative exchange between sender and recipient". In the PIM workshop, people considered the notion of PIM differently.

Information here indicates information which affects "change in our lives and in the lives of others". The information we receive influence the actions we take and the choices we make. For example, meeting information sending to us specifies which room we will go to attend the meeting.

We also send information out to influence changes. We send information about the clothes we choose to wear, the car we choose to drive, and in the way we choose to act. We send information (often more than we intend) with every sentence we speak or write, which are all with respect to the information we send.

The original definition of information is not enough to describe information. We send information not only for exchanging but also for serving our own purposes. Certainly one of these purposes is to be helpful and inform others. But we also send information to persuade, convince, impress and, sometimes, to deceive. Conclusively, the definition "affects which change in our lives and in the lives of others" describes information here better.

Information Item An information item is a packaging of information. Examples of information items includes: paper document, electronic documents and other files, email messages, web papers, XML element, tuples in a database table and references to any of the above. 
Items encapsulate information and keep information persistent. Items can be created, stored, moved, entitled, described, copied, distributed, deleted, modified, etc. In order to emphasize that an information item can be manipulated, some might replace "information item" to "information object".

Information form is an important factor relative to information item. Information items are supported by applications working on computers. Different information items are stored and managed by different applications. Email clients manage emails, file system manages documents, and web browser works for information from World Wide Web. Every application has its own way to determine information form so that applications can name, move, copy, delete, organize and assign properties to information items uniformly.

The size of an information item varies and is determined by the application which stores the information item. The size of a document which is stored with compressing technology is smaller than the size when it is stored without compression technology. Different technologies storing the same information item result in diversity of information size. The size is influenced by who is reading it. For example, file system treats a contract as an information item but the company who owns this contract treats the individual entity in the contract as an information item.

An important task of an information item is to mediate people and the world. People read weather information on the web site in order to know what they had better to wear. Students send emails to submit assignments to professors. Information items play important role in daily-life.

Apart from mediation, information items have other tasks. People can gather information items earlier than when information items are used. Or, information is accumulated for no such reason which appears later until most of information is collected.

Personal Information Personal information is the information people keep for their own personal use; it is the information about a person but possibly kept by and under the control 
of others; it is the information experienced by a person even if this information remains outside a person's control.

Personal Information Management In a computing field, Personal Information Management (PIM) is "an umbrella term used to describe the collection, storage, organization and retrieval of items of digital information (e.g. email, files, appointments, reminders, contacts, bookmarks) by an individual in their personal computing environment" [31]. Note that, we only consider the definition of PIM in the computing field in this thesis.

\subsection{Dataspace}

Dataspace is a new data management architecture where heterogeneous data co-exists without conducting semantic integration in advance. In the imagination of Dataspace, basic functionalities as keyword search can be provided over data residing at all sources, regardless of how integrated they are. Moreover, it allows advanced services by performing further semantic integration whenever needed. The main characteristic of Dataspace is: Pay-as-you-go fashion. Pay-as-you-go means that before conducting semantic integration, designers do not need to create precise schema for it, and the schema is summarized gradually whenever needed. Dataspace is different from what traditional data management architecture requires. The traditional data management architecture requires data to conform to one single schema. A typical case is that attributes and types of attributes are predefined and data inserted in the relation should conform to the limitations of attributes.

Data management systems in Dataspace vision do not predefine schemas and do not conduct semantic integration. Instead, they provide services when data is co-existed while not semantically integrated yet. They extract semantic information and conduct semantic integration after users require it. The benefit of co-existing data is that it took less effort on unify data so that it is able to provide services for users sooner. 


\begin{tabular}{|c|c|c|}
\hline & Dataspace & $\begin{array}{c}\text { Traditional Data Management } \\
\text { Architecture }\end{array}$ \\
\hline Schema & Flexible & Predefined and fixed \\
\hline Semantic Integration & Required whenever needed & Required before any service is provided \\
\hline Data Control & Best effort & Well Controlled \\
\hline
\end{tabular}

Table 2.1: Comparison of Dataspace and traditional data management architecture

We summarize the differences of Dataspace and traditional data management architecture in Table 2.1. We compare them from three different respects: Schema, Semantic Integration and Data Control. Dataspace does not require to predefine schema and traditional data management architecture requires to predefine schemas; the former asks for co-existent data and the latter asks for semantically integrating data; the former does not fully control data while the latter controls data very well.

Dataspace is introduced to deal with heterogeneous data sources. Heterogeneous data sources have a great number of data formats. To deal with such data sources in traditional data management architecture is impractical. Traditional data management architecture requires a design to provide a schema into which all data can be fitted. To summarize a schema which suits all heterogeneous data sources is very difficult and not practical. As a result, to deal with heterogeneous data sources, we should choose Dataspace vision.

Personal Information Management is one typical scenario of Dataspace. Personal information is heterogeneous and we should manage such information in Dataspace vision. Such PIM systems have the following characteristics: they do not predefine schema for personal information; they collect data and make it co-existent; they summarize semantic information from co-existent data gradually. As indicated in [7], there are two rules for PIM systems in Dataspace: "Firstly, a PIM tool must enable accessing all the information on the desktop, and not just an explicitly chosen subset. Secondly, most of the time the 
system will have to provide best-effort results and tighter integrations will be created only in cases where the benefits clearly outweigh the investment".

\subsection{Data Model}

What data model suits the Dataspace vision is quite an important issue. We give a brief description of significant data models involved in data management systems. We then conclude what characteristics a data model should have in order to be applied in Dataspace vision.

Early data management systems were based on the relational data model. They provide a single global schema by deploying tightly-coupled solutions. The relational model is simple to understand and it is easy to access. However, relational models cannot represent all kinds of data, because there are always special types of data which require special forms of representation. To overcome this limitation, object-oriented model were adopted in data management. In 1990's, Internet and web technologies were introduced and with their development, semi-structured data and unstructured data are involved in data management. The interest of data management shifted from managing structured data into managing semi-structured/unstructured data.

In the mid 90s, Tsimmis [2] was developed as the first data management system based on a semi-structured data model. A semi-structured data model is a model which can present data and the schema together. T'simmis is built based on a common objects model, called Object Exchange Model (OEM), OEM has the form < Label of data object, Type of the data object, Value of the data object $>$. The model is simple, flexible and expressive. However, it only presents properties of unstructured data objects. Apparently, only containing property information of unstructured data objects is too limited. After Tsimmis, several integration systems such as Haystack[25] and Semex[27] were developed based on XML and RDF, which all belong to semi-structured data model. Semi-structured data 
model is considered as a flexible data model which is able to present both data information and schema information.

The relational model and object-oriented model predefine schemas for data while semistructured data models reduce the effort of predefining schema. Semi-structured data models provide flexible formats for presenting data. They get rid of the separation between data and schema. Data model in Dataspace should be a flexible data model which can represent different kinds of data sources. Hence, semi-structured data models suit for Dataspace better.

\subsection{Storage}

This section introduces storage approaches which could be applied to store heterogeneous data sets. The normal data storage schema is the Horizontal Schema. We first discuss the usage of the Horizontal Schema in storing heterogeneous data sets. We show that the Horizontal Schema is not good for storing heterogeneous data sets. Secondly, we discuss the Vertical Schema, which is considered to be a better way to store heterogeneous data sets. Thirdly, we present current storage strategies in commercial RDF stores and most of them are based on the Vertical Schema. The last part of this section presents a physical storage format, interpreted format, which is used to store sparse data sets. We consider that sparse data sets and heterogeneous data sets have common characteristics and the interpreted format suits heterogeneous data sets as well. We give examples for all storage strategies based on the example data shown in Figure 2.1.

\subsubsection{Horizontal Schema}

The Horizontal Schema approach is to map the entity set to a table, and the attributes of the table contain all the attributes for all entities. That is, any entity among the entity set can find all its attributes in the table. Table 2.2 shows how to store heterogeneous data set. 


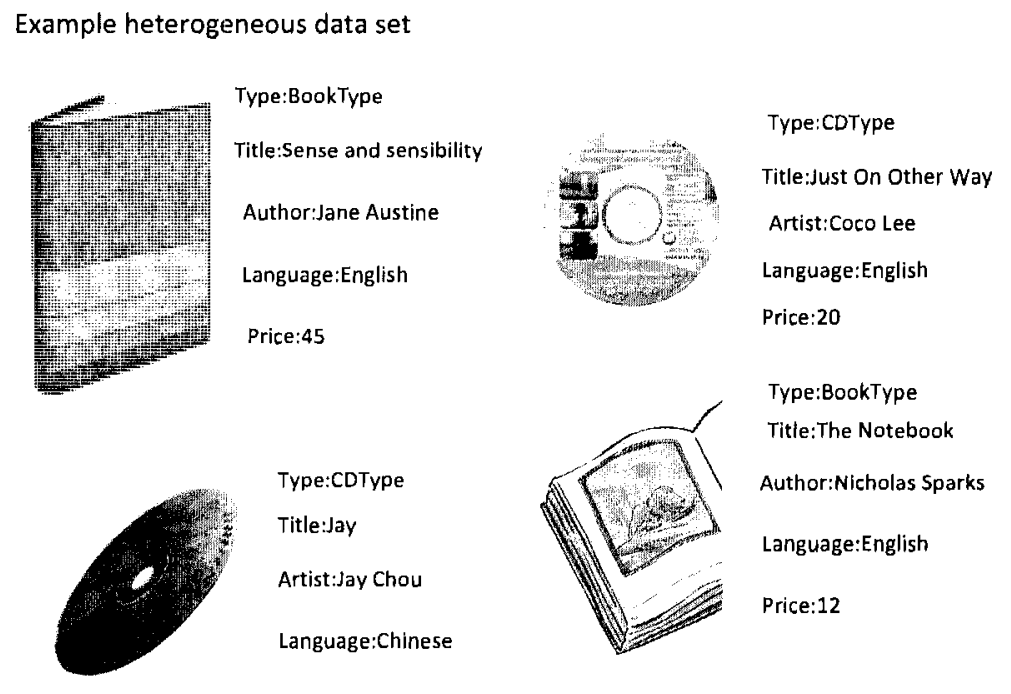

Figure 2.1: Example heterogeneous data set

\begin{tabular}{|l|l|l|l|l|l|l|}
\hline Entity ID & Type & Title & Author & Artist & Language & Price \\
\hline 1 & BookType & $\begin{array}{l}\text { Sense and } \\
\text { Sensibility }\end{array}$ & Jane Austine & Null & English & 45 \\
\hline 2 & CDType & Jay & Null & Jay Chou & Chinese & Null \\
\hline 3 & BookType & The Notebook & Nicholas Sparks & Null & English & 12 \\
\hline 4 & CDType & Just No Other Way & Null & Coco Lee & English & 20 \\
\hline
\end{tabular}

Table 2.2: Horizontal Schema 
The advantages of the Horizontal Schema are as follows: it is the most natural and straightforward approach to store data; all entities with their attributes can be stored in one table; it is easy to manipulate data. The Horizontal Schema approach can be used to store heterogeneous data set.

As we can see in Table 2.2, attributes of the table contain attributes of all entities, the consequence of which is there are null values in the table. If, in heterogeneous data set, attributes of entities cannot be predicted and entities do not have many common attributes, the table will be predominantly filled with a lot of nulls. The result is that the table space is occupied by a lot of useless null values and it is slow to scan in such tables.

\subsubsection{Vertical Schema}

In the Vertical Schema, there are only three columns in the table, which are Subject, Attribute and Attribute Value. Here, Table 2.3 shows how to store heterogeneous data set in Table 2.2 in Vertical Schema.

The benefit of the Vertical Schema for storing heterogeneous data set is obvious. It is very flexible. No matter how many different attributes there are, the vertical Schema can handle it well and it will not waste any space for nulls as the Horizontal Schema does.

The Vertical Schema brings some problems as well. The first problem is the data type problem; that is, there is only one column to store attribute values with different data types. All types of attributes are degraded to be one type. The second problem is that operations on data become very complicated. Information of an entity is decomposed and stored in several tuples. To query information of an entity, we need to search for those tuples and combine them. As a result, we need more operations than we have with the Horizontal Schema. 


\begin{tabular}{|l|l|l|}
\hline EntitylD & Attribute & Attribute Value \\
\hline 1 & Type & BookType \\
\hline 1 & Title & Sense and Sensibility \\
\hline 1 & Author & Jane Austine \\
\hline 1 & Language & English \\
\hline 1 & Price & 45 \\
\hline 2 & Type & CDType \\
\hline 2 & Title & Jay \\
\hline 2 & Artist & Jay Chou \\
\hline 2 & Language & Chinese \\
\hline 3 & Type & BookType \\
\hline 3 & Title & The Notebook \\
\hline 3 & Author & Nicholas Sparks \\
\hline 3 & Language & English \\
\hline 3 & Price & 12 \\
\hline 4 & Type & CDType \\
\hline 4 & Title & Just No Other Way \\
\hline 4 & Artist & Coco Lee \\
\hline 4 & Language & English \\
\hline 4 & Price & 20 \\
\hline
\end{tabular}

Table 2.3: Vertical Schema 


\subsubsection{Approaches in RDF Store}

RDF is basically a Triple Format. Current RDF Stores mostly applied or modified Horizontal Schema and Vertical Schema in their own store designs. Those approaches include: RDF Triples Table, Property-Clustered Table and Property-Class Table. We present those approaches in following subsections.

\subsubsection{RDF Triples Table}

Many commercial RDF stores such as Jena [34], Oracle [23], Sesame [22], and 3store [27] use the Vertical Schema in their underlying storage and the Vertical Schema is titled as RDF Triples Table in RDF stores. Some RDF stores choose to store shortened versions or keys. Oracle and Sesame map string Uniform Resource Identifiers (URIs) (URIs usually are long strings) to integer identifiers so the data is normalized into two tables, one Triples table using identifiers for each value, and one mapping table that maps the identifiers to their corresponding strings. It is called as dictionary encoding strings. 3store does something similar. The identifiers are created by applying a hash function to each string. Jena prefers to just dictionary encode the namespace prefixes in the URIs and only normalizes the particularly long strings into a separate table.

\subsubsection{Property Tables}

Property-Clustered Table Such tables contains clusters of properties that tend to be defined together, along with the left-over triples that are not stored in this property table stored in left-over Triples table. Note that the term property in RDF store is the same as attribute in Horizontal Schema and Vertical Schema. Multiple Property Tables with different clusters of properties may be created; however, a key requirement for PropertyClustered Table is that a particular property can only appear in at most one property table. An example is shown in Table 2.4 . 


Property Table
\begin{tabular}{|l|l|l|l|}
\hline Entity ID & Type & Title & Language \\
\hline 1 & BookType & Sense and Sensibility & English \\
\hline 2 & CDType & Jay & Chinese \\
\hline 3 & BookType & The Notebook & English \\
\hline 4 & CDType & Just No Other Way & English \\
\hline
\end{tabular}

Left-Over Triples
\begin{tabular}{|l|l|l|}
\hline EntityID & Attribute & Attribute Value \\
\hline 1 & Author & Jane Austine \\
\hline 1 & Price & 45 \\
\hline 2 & Artist & Jay Chou \\
\hline 3 & Author & Nicholas Sparks \\
\hline 3 & Price & 12 \\
\hline 4 & Artist & Coco Lee \\
\hline 4 & Price & 20 \\
\hline
\end{tabular}

Table 2.4: Property Clustered Table

Property-Class Table Property-Class Table exploits a specific property (this property had better appear in almost all entities) of entities to cluster similar sets of entities together in the same table. A property may exist in multiple Property-Class Tables. Example is shown in Table 2.5. In example, type property is selected to be the key property to classify triples.

The most important advantage of the introduction of Property Tables is that they can ease searching operations which searches data in Property Tables.

The disadvantage of Property Tables approaches is that they require property clustering to be carefully done to create Property Tables that are not too wide, while still being wide enough to answer most queries directly. 


Class: BookType
\begin{tabular}{|l|l|l|l|}
\hline Entity ID & Title & Language & Price \\
\hline 1 & Sense and Sensibility & English & 45 \\
\hline 3 & The Notebook & English & 12 \\
\hline
\end{tabular}

Class:CDType
\begin{tabular}{|l|l|l|}
\hline Entity ID & Title & Language \\
\hline 2 & Jay & Chinese \\
\hline 4 & Just No Other Way & English \\
\hline
\end{tabular}

Left-Over Triples

\begin{tabular}{|l|l|l|}
\hline EntitylD & Attribute & Attribute Value \\
\hline 1 & Author & Jane Austine \\
\hline 2 & Artist & Jay Chou \\
\hline 3 & Author & Nicholas Sparks \\
\hline 4 & Artist & Coco Lee \\
\hline 4 & Price & 20 \\
\hline
\end{tabular}

Table 2.5: Property Class Table

\subsubsection{Vertically Partitioned Approach}

Research work in [29] proposes storing RDF data using the Vertically Partitioned Approach table, the definition of which is as follows: the triples "rewritten into n two column tables where $\mathrm{n}$ is the number of unique properties in the data. In each of these tables, the first column contains the subjects that define that property and the second column contains the object values for those subjects". For example, the triples table from Table 2.3 would be stored as in Table 2.6. As claimed in [29], queries over storages using Vertically Partitioned Approach have the same efficiency as queries over storages using Property Tables.

\subsubsection{Interpreted Format}

Interpreted format was introduced to store spare data set by BeckMann et al. [1]. The interpreted format stores a list of attribute-value pairs.

Figure 2.2 shows an entity stored vertically and as an interpreted tuple separately. Note that both of them store "attribute, value" pairs. The primary distinctions are: in interpreted 


Type
\begin{tabular}{|l|l|}
\hline 1 & BookType \\
\hline 2 & CDType \\
\hline 3 & BookType \\
\hline 4 & CDType \\
\hline
\end{tabular}

Title

\begin{tabular}{|l|l|}
\hline 1 & Sense and Sensibility \\
\hline 2 & Jay \\
\hline 3 & The Notebook \\
\hline 4 & Just No Other Way \\
\hline
\end{tabular}

Language

\begin{tabular}{|l|l|}
\hline 1 & English \\
\hline 2 & Chinese \\
\hline 3 & English \\
\hline 4 & English \\
\hline
\end{tabular}

Author

\begin{tabular}{|l|l|}
\hline 1 & Jane Austine \\
\hline 3 & Nicholas Sparks \\
\hline
\end{tabular}

Artist

\begin{tabular}{|l|l|}
\hline 2 & Jay Chou \\
\hline 4 & Coco Lee \\
\hline
\end{tabular}

Table 2.6: Vertically Partitioned Approach

format, all the pairs are stored in a single tuple so there is no need to tie "attribute, value" pairs together with a common tuple id or reconstruct the tuple during query evaluation; in interpreted format, the attributes are collected together as one object, and in contrast, the vertical entity is a set of independent tuples that can be organized (or clustered) in any order.

The spare data set and heterogeneous data set has common characteristic which is that both of them have diverse attributes. We believe that interpreter format suits heterogeneous data set better than Vertical Schema.

\subsection{Query}

One goal of our thesis is to provide structured query which can return accurate query results; and to provide a keyword search which combines the nice features of structured query and traditional keyword search. According to the goal, we research current Triple Query 
Vertical Storage of an Entity

\begin{tabular}{|l|l|l|}
\hline EntitylD & Attribute & Attribute Value \\
\hline 1 & Type & BookType \\
\hline 1 & Title & Sense and Sensibility \\
\hline 1 & Author & Jane Austine \\
\hline 1 & Language & English \\
\hline 1 & Price & 45 \\
\hline
\end{tabular}

Interpreted of an Entity

1 Type BookType Title Sense and Sensibility Author Jane Austine Languge English Price 45

Figure 2.2: Store an Entity into Interpreted Format

Languages and indexing technologies.

\subsubsection{Triple query language}

Triple query language is the same term as RDF query language. We discuss triple query languages from the perspective of query language properties. There are 5 properties that a query Language should follow. They are expressiveness, closure, adequacy, orthogonality, adequacy and safety. Expressiveness indicates how powerful queries can be performed in a given language. The closure property requires that the results of an operation are again elements of the data model. A query Language is called adequate if it uses all concepts of the underlying data model. The orthogonality of a query language requires that all operations may be used independently of the usage context. A query language is considered safe if every query that is syntactically correct returns a finite set of results.

RQL [17], SeRQL [18], TRIPLE [20], RDQL [19], N3 [21, 22] are most popular RDF query languages currently. Most of them support querying over RDF. Among them, RQL is OQL-like, RDQL is SQL-like language, TRIPLE applies F-Logical expression [23], N3 provides a text-based syntax for RDF and SeRQL is a querying and transformation 
language loosely based on RQL, RDQL and N3.

We summarize those languages in Table 2.7. RQL cannot return graph which is an element in RDF model and its semantics is not completely compatible with the RDF semantics, so RDL does not fulfill closure and adequacy. TRIPLE does not return graph as well and it allows recursion. In addition, it does not support RDF model directly while it maps RDF to its own data model. During the mapping, some data is lost. Therefore, TRIPLE is not closed, adequate and safe. SeRQL can return graph and variable/value bindings and it supports RDF model. However, it provides recursion which might be infinite. So, SeRQL is closed, adequate but unsafe. N3 only does not fulfill orthogonality. RDQL only returns variable/value bindings and it does not interpreted RDF schema information. Hence, RDQL is not closed and adequate. Since we are not going to focus on features of RDF in this thesis, we do not compare features specific for RDF. Among all query languages mentioned above, SeRQL is unification of best practices from varying query languages and is delivering a light-weight yet expressive query language for RDF that addresses practical concerns [24]. Hence, for a user, SeRQL is the best choice. As we have observed, no triple query language fulfills all language properties.

\subsubsection{Indexing approaches}

Traditional index approaches aims at indexing content for a document. They mainly deal with unstructured data. Recently, many researchers had started to pay more attention to indexing structured or semi-structured data inside a document. The three bodies of work of indexing on structured or semi-structured data are indexing XML document, keyword queries in relational databases and indexing heterogeneous data.

Indexing approaches in XML document can be classified into three kinds: indexing on value, indexing on structure and indexing on both value and structure. The first kind (e.g., 


\begin{tabular}{|c|c|c|c|c|c|c|c|}
\hline \multicolumn{3}{|c|}{ Features Query } & RDQL & Triple & SeRQL & N3 & $\mathrm{RQL}$ \\
\hline \multirow{9}{*}{$\begin{array}{l}E \\
X \\
P \\
R \\
E \\
S \\
S \\
I \\
O \\
N\end{array}$} & \multirow[b]{2}{*}{ Graph } & $\begin{array}{c}\text { Path } \\
\text { Expression }\end{array}$ & $Y$ & $Y$ & $Y$ & Y & $\mathrm{Y}$ \\
\hline & & $\begin{array}{l}\text { Optional } \\
\text { Expression }\end{array}$ & - & - & $Y$ & - & - \\
\hline & \multirow[t]{3}{*}{ Relational } & $\begin{array}{c}\text { Basic } \\
\text { algebraic } \\
\text { operations }\end{array}$ & $Y$ & $Y$ & $Y$ & $Y$ & $\mathrm{Y}$ \\
\hline & & Union & - & $Y$ & - & $Y$ & $Y$ \\
\hline & & Difference & - & - & - & - & $Y$ \\
\hline & \multirow{2}{*}{$\begin{array}{l}\text { Aggregati } \\
\text { on } \\
\text { and } \\
\text { Grouping }\end{array}$} & Aggregation & - & - & - & $Y$ & $Y$ \\
\hline & & Grouping & - & - & - & - & - \\
\hline & \multicolumn{2}{|c|}{ Recursion } & - & $Y$ & - & $Y$ & - \\
\hline & \multicolumn{2}{|c|}{$\begin{array}{l}\text { Other features specific } \\
\text { for RDF }\end{array}$} & & 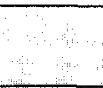 & $\because$ & & $\therefore$ \\
\hline \multicolumn{3}{|c|}{ Closure } & $\mathrm{N}$ & $\mathrm{N}$ & Y & $Y$ & $\mathrm{~N}$ \\
\hline \multicolumn{3}{|c|}{ Adequacy } & $\mathrm{N}$ & $N$ & $Y$ & $\mathbf{N}$ & $\mathbf{N}$ \\
\hline \multicolumn{3}{|c|}{ Orthogonality } & $Y$ & $Y$ & $Y$ & $Y$ & $Y$ \\
\hline \multicolumn{3}{|c|}{ Safety } & $Y$ & $\mathrm{~N}$ & $N$ & $Y$ & $Y$ \\
\hline
\end{tabular}

Table 2.7: Language Comparison 
$[36,37,38])$ indexes text values and at the same time encodes parent-child and ancestordescendent relationships. The second kind (e.g., [39, 40]) only indexes on schemas of a XML document. The third kind (e.g., [41]) combines on indexes on structure and on text.

Several works (e.g., [42]) have considered keyword queries on relational databases. They either require building the join-network at run-time or recomputed and index distance between elements. Those approaches are usually expensive.

Works such as SphereSearch [43], Kite [44] study search across heterogeneous data by first conducting data transformation or integration. However, their indexes works are based on that the data are tightly coupled. Research work in [45] took an approach which indexes heterogeneous data even if the data are only loosely coupled.

Among all index approaches, works in [45] perfectly fit Dataspace vision because it indexes heterogeneous data and it does not require semantic integration in advance. Hence, we took this index approach in building the keyword search function for our system.

\subsection{Current PIM system and our PIM system}

\subsubsection{A brief survey of PIM systems}

MyLifeBites [34] It is a system to explore the use of SQL to store all personal information found in PCs. The main idea is to make everything in Database. In MyLifeBits, all captured entities explored to the users are defined as "Items". MyLifeBites stores attributes and text content of all entities into database. There are Links table to store the relationship among different items as well. Since all information is in database, MyLifeBits has full control of data and operations to personal information stored in databases are possible.

WinFS [51] Developed by Microsoft, it is a system to store and manage structured, semistructured as well as unstructured data. The core idea of WinFS is to store metadata and data in relational databases whenever possible. WinFS provides well defined schema for 
any type of information so that any type of information can be stored in databases properly. WinFS also defined relationships between individual data items. Apart from predefined type schemas and relationships, WinFS allows users to customize attributes and relationships of data items.

Haystack [28] It is developed based on semi-structured data model, RDF Model, which offers the right balance of rich structure and flexible schema allowing naive end users to record information in different forms. In Haystack, the RDF Model presents data and cross-domain relationship information. Though Haystack applied a flexible data model, it sill aimed at conducting semantic integration.

Semex [27] It predefined schema for integrating personal information and model domain knowledge in RDF. Its significant research works include: reference reconciliation which is a work for semantic integration, answering structured queries on unstructured data and its indexing technology [45].

Imemex [33] It is the first PIM system which was developed in Dataspace. It explored a model IDM [47] claimed as a model which can present all personal information. The definition of IDM is complex to understand and building systems on it is not easy. As declaimed in [46], IDM is not a really flexible model.

Our PIM System Comparing to other PIM systems, our PIM system applies a more flexible data model, the PIM model, which can represent various data models easily and powerfully and makes representation of heterogeneous data easy by reducing the effort of predefining the schema. In addition, our system effectively and efficiently collect personal information to make it co-existent so that basic functionalities such as keyword search and structured query are provided sooner. Our system is easy to extend so that when users ask for more advanced services, semantic integration is able to conducted. 


\subsubsection{Summary}

We summarize PIM systems discussed above into Table 2.8. We have observed: modeling strategy evolves from schema fist to schema later; modeling approaches change on the purpose of eliminating the need to predefine schemas; information extraction becomes more specific and complex; the vision of PIM systems follow is tempted to be replaced by Dataspace; data storages have been involved are decided by data models of PIM systems.

Our systems adopts a novel semi-structured model PIM Model to model data with a simple schema which eliminates the need to predefine schemas, develops decomposers to decompose structured, unstructured and semi-structured data sources, and fulfills Dataspace architecture to provide services, explores storage for PIM Model. In addition, we develop structured query and keyword search functions to query personal information. Detailed design and implementation of our system are discussed in the Chapter Model and Implementation. 


\begin{tabular}{|c|c|c|c|c|c|}
\hline & \multicolumn{2}{|l|}{ Models } & \multirow[t]{2}{*}{ Information Extraction } & \multirow{2}{*}{$\begin{array}{l}\text { Data } \\
\text { Management } \\
\text { Architecture }\end{array}$} & \multirow[t]{2}{*}{ Storage } \\
\hline & $\begin{array}{l}\text { Modeling } \\
\text { strategy }\end{array}$ & $\begin{array}{l}\text { Modeling } \\
\text { Approaches }\end{array}$ & & & \\
\hline MyLifeBites & $\begin{array}{l}\text { Schema } \\
\text { first }\end{array}$ & $\begin{array}{l}\text { Object-Oriented } \\
\text { Model }\end{array}$ & $\begin{array}{l}\text { Property Information } \\
\text { and content of } \\
\text { resources }\end{array}$ & DBMS & $\begin{array}{l}\text { Relational } \\
\text { database }\end{array}$ \\
\hline WinfS & $\begin{array}{l}\text { Schema } \\
\text { first }\end{array}$ & $\begin{array}{l}\text { Object-Oriented } \\
\text { Model }\end{array}$ & $\begin{array}{l}\text { Property information } \\
\text { and Relationships } \\
\text { between information } \\
\text { items }\end{array}$ & DBMS & $\begin{array}{l}\text { Relational } \\
\text { database }\end{array}$ \\
\hline Haystack & $\begin{array}{l}\text { Schema } \\
\text { first }\end{array}$ & $\begin{array}{l}\text { Semi-structured } \\
\text { Model }\end{array}$ & $\begin{array}{l}\text { Property information } \\
\text { and Relationships } \\
\text { between information } \\
\text { items }\end{array}$ & DBMS & RDF \\
\hline Semex & $\begin{array}{l}\text { Schema } \\
\text { first }\end{array}$ & $\begin{array}{l}\text { Semi-structured } \\
\text { model }\end{array}$ & $\begin{array}{l}\text { Property information and } \\
\text { Relationships } \\
\text { between information } \\
\text { items }\end{array}$ & DBMS & RDF \\
\hline Imemex & $\begin{array}{l}\text { Schema } \\
\text { later }\end{array}$ & Logical Model & $\begin{array}{l}\text { Property information } \\
\text { Relationships between } \\
\text { information } \\
\text { items, structured } \\
\text { information inside } \\
\text { structured and } \\
\text { Semi-structured data } \\
\text { sources }\end{array}$ & Dataspace & $\begin{array}{l}\text { Relational } \\
\text { database }\end{array}$ \\
\hline $\begin{array}{l}\text { Our PIM } \\
\text { system }\end{array}$ & $\begin{array}{l}\text { Schema } \\
\text { later }\end{array}$ & PIM Model & $\begin{array}{l}\text { Property information } \\
\text { Relationships between } \\
\text { information } \\
\text { items, structured } \\
\text { information inside } \\
\text { structured and } \\
\text { Semi-structured data } \\
\text { sources }\end{array}$ & Dataspace & $\begin{array}{l}\text { Berkeley } \\
\text { DB }\end{array}$ \\
\hline
\end{tabular}

Table 2.8: Comparison of different PIM Systems 


\section{Chapter 3}

\section{PIM Model}

This chapter introduces a semi-structured model, called PIM Model. The main feature of the PIM Model is its flexibility, which eases the effort to predefine schemas before storing heterogeneous data. This chapter is structured as follows: definitions of the PIM Model, representation of various data models with the PIM Model, formation of a graph and features of the PIM Model.

\subsection{Definition}

This section presents definitions of the PIM Model. We assume the existence of the following pairwise disjoint sets:

- primitive data types $\mathrm{T}=\{$ String, Integer, Real, Date, ID $\}$;

- a set $\mathrm{P}$ of property names;

- a set of property values $V=S \cup I \cup R \cup D \cup O$ where $S$ is a set of strings, $I$ is a set of integers, $\mathrm{R}$ is a set of real numbers, $\mathrm{D}$ is a set of dates, and $\mathrm{O}$ is a set of IDs;

- a set $\mathrm{C}$ of class names. 


\subsubsection{Information Item}

The same as defined in Chapter 2, an information item is a package of information such as a file, a folder, a relational tuple, an XML element and a web page.

An information item is an expression of the form:

$$
o\left[\left(\left(t_{1}: p_{1}\right), v_{1}\right), \ldots,\left(\left(t_{n}: p_{n}\right), v_{n}\right)\right]
$$

where:

- $o \in O$ is an instance of type ID representing the information item;

- $p_{i} \in P$ is a property of $o$ for $1 \leq i \leq n$;

- $t_{i} \in T$ is the data type of $p_{i}$ for $1 \leq i \leq n$;

- $v_{i} \in V$ or $v_{i} \subseteq V$ is the value of $p_{i}$ for $1 \leq i \leq n$;

- if $t_{i} \neq I D$, then $p_{i}$ is an attribute; otherwise, $p_{i}$ is a relationship;

In an information item of the form (3.1), we call $\left(o,\left(t_{i}: p_{i}\right), v_{i}\right)$ a triple. A triple has subject component $o$, property component $p_{i}$ and object component $v_{i}$.

For example, consider a book called Chinese Dishes. It has attributes name, page, author; their corresponding values are "Chinese Dishes", 50 and "Wang Ming"; their data types are String, Integer and String. Chinese Dishes has two chapters Soup and Noodle. It is represented as an information item as follows:

chinesedishes[

((String:name), "Chinese Dishes"),

((Integer:page), 50),

((String:author), "Wang Ming"),

((ID:hasChapter), \{soup, noodle $\})$,

]

where chinesedishes, soup and noodle are ids of book Chinese Book, Soup and Noodle. 


\subsubsection{Information Item Classes}

An information item can belong to various information item classes. The expression used to specify this is of the form:

$$
c_{1}, \ldots, c_{n} o
$$

where $o$ is the id of an information item and $c_{i} \in C$ is an information item class of $o$ for $0 \leq i \leq n$.

For example, let Chinese_Recipes and Chinese_Book be two classes. To assign chinesedishes to be an instance of these two classes, we can use the following declaration:

Chinese_Recipes, Chinese_Book chinesedishes

To present an information item together with its class information, we can combine (3.1) and (3.2) into the following expression:

$$
c_{1}, \ldots, c_{n} \circ\left[\left(\left(t_{1}: p_{1}\right), v_{1}\right), \ldots,\left(\left(t_{n}: p_{n}\right), v_{n}\right)\right]
$$

For example, we can combine the two declarations above using expression into the following declaration:

Chinese_Recipes, Chinese_Book chinesedishes[

((String:name), "Chinese Dishes"),

((Integer:page), 50),

((Integer:author), "Wang Ming"),

((ID:hasChapter), \{soup, noodle $\}$ ),

]

Consider a book called Wine Book. It has 36 pages and its author is " $\mathrm{Li} \mathrm{Xu".} \mathrm{It} \mathrm{belongs}$ to classes English_Book, Beverage. Wine Book can be presented as follows:

English_Book, Beverage winebook[ 


\section{((String:name), "Wine Book"), \\ ((Integer:page), 36), \\ ((String:author), "Li Xu"), \\ ]}

where winebook is the id of book Wine Book.

Consider a book called Western Food. It has 54 pages and its author is "Jimmy Hanks". It belongs to classes Book and Western_Recipes. Western Food can be presented as follows:

Book, Western_Recipes westernfood[

((String:name), "Western Food"),

((Integer:page), 54),

((String:author), "Jimmy Hanks"),

]

where westernfood is the id of book Western Food.

\subsubsection{Class Hierarchy}

A class can have subclasses. Subclasses of a class can be declared using an expression of the form:

$$
\text { c subsumes } c_{1}, \ldots, c_{n}(n \geq 0)
$$

where $c$ and $c_{i} \in C$ are classes for $0 \leq i \leq n$.

For example, to specify that class Book has two subclasses Chinese_Book and English_Book, we can use the following declaration:

Book subsumes Chinese_Book, English_Book

Another example is that class Cook has two subclasses Food and Beverage. Class Food has subclasses Chinese_Recipes and Western_Recipes. It is presented as:

Cook subsumes Food, Beverage

Food subsumes Chinese_Recipes, Western_Recipes

With information item classes, an information item can belong to multiple classes and 


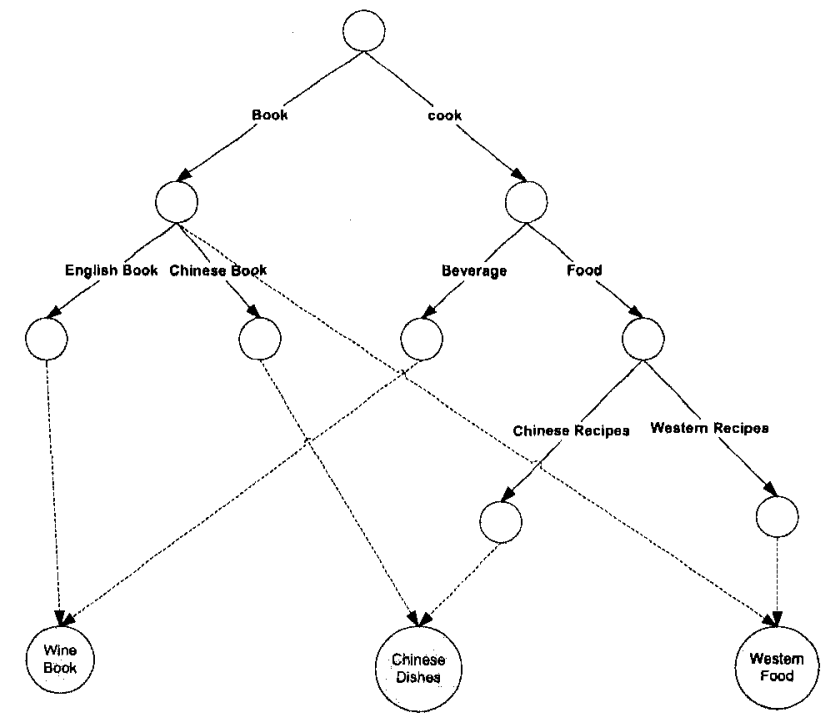

Figure 3.1: Example classes structure

classes can be organized hierarchically. The Figure 3.1 shows the classes structure of book chinesedishes, winebook and westernfood.

\subsection{Representation of other models with PIM Model}

The flexibility of the PIM Model allows it to represent other models. We present ways of converting information from other data models to the PIM Model.

\subsubsection{File System to PIM Model}

In a file system, there are mainly two kinds of information item: folder and file. 


\subsubsection{Representation of a folder}

Folder information item has two kinds of information: attribute information and relationship information.

Attributes of a folder information item are name, path, size and created time. Their types are String, String, Integer and Date.

A folder has relationship information that represents its relationships to other folders and files in it. A folder can have subfolders and we define relationship between a folder and its subfolders as "ID:subFolder". A folder can contain files and we define relationship between a folder and its files as "ID:containsFile".

Assume that a folder information item $\pi$ has attributes: name with value $v_{\text {name}}$; path with value $v_{\text {path }}$; size with value $v_{\text {size }}$; created time with value $v_{\text {createdtime. Assume that } \pi}$ has a group of files $\pi_{1}, \ldots, \pi_{n}$ for $n \geq 0$ and a group of subfolders $\sigma_{1}, \ldots, \sigma_{m}$ for $m \geq 0$. The rule to represent a folder's information has the following form:

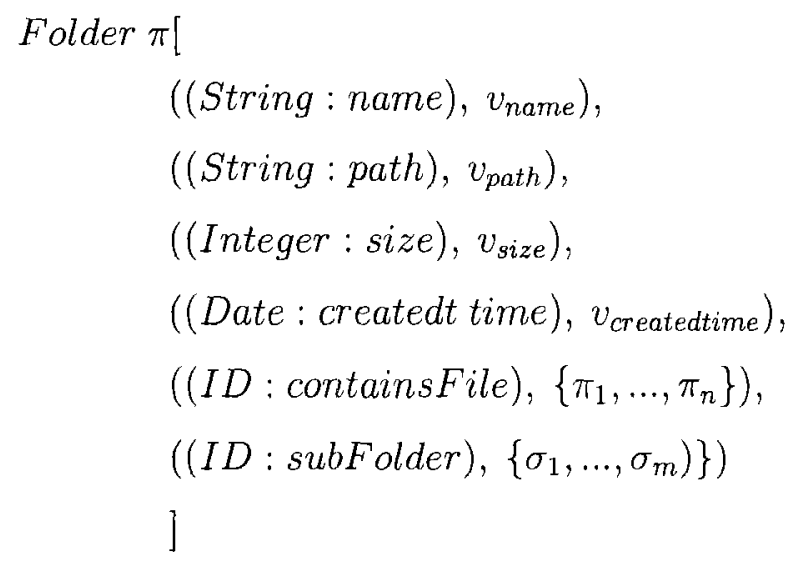

Figure 3.2 shows attribute information of folder Cook Book and Figure 3.3 shows relationship information of Cook Book. Cook Book has subfolders Dish and Drink, and contains a file Paper. We can represent attribute information and relationship information of the folder as follows:

Folder cookbook[ 


$\begin{aligned} & \text { Namé } \\ & \text { Date taken Took Book }\end{aligned}$
\[ \begin{array}{l}\text { Date created 2008/11/18 17:16 } \\ \text { Size 19.2 KE } \\ \text { Folders: Cook Book }\end{array} \]

Figure 3.2: A folder's attribute information

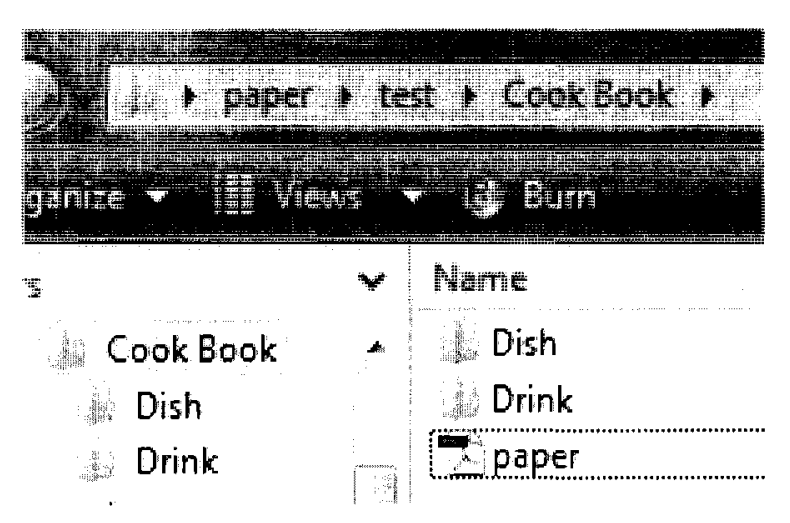

Figure 3.3: A folder's relationship information 
((String:name), "Cook Book"),

((String:path), "C:/Users/Qian/Desktop/paper/test/Cook Book"),

((Integer:size), 129000),

((Date:created time), "2008/11/18 17:16"),

((ID:containsFile), \{paper\})

((ID:subFolder), \{dish, drink\}),

]

where cookbook, paper, dish and drink are ids of Cook Book, Paper, Dish and Drink.

\subsubsection{Representation of a file}

A file information item has two kinds of information: attribute information and relationship information.

Attributes of a file are: name of type String; size of type Integer; last modified time of type Date; Type of type String; extensionName of type String; path of type String.

Relationship of a file is the relationship between the file and the information items inside the file, such as relationship between an XML file and the root node of the XML document. We define such relationship as "ID:hasItem".

Assume that a file $\pi$ has attributes: name with value $v_{\text {name }}$; size with value $v_{\text {size }}$; last modified time with value $v_{\text {lastmodifiedtime }}$; type with value $v_{\text {type }}$; extensionName with value $v_{\text {extensionName }}$; path with value $v_{\text {path }}$. Also assume that $\pi$ has a group of information items $\pi_{1}, \ldots, \pi_{n}$ for $n \geq 0$ inside it. Information of $\pi$ can be listed as:

File $\pi$ [ 
Type: XML Document

Size: $16.6 \mathrm{kB}$

Date modified: $2008 / 11 / 1017: 52$

Figure 3.4: Example file

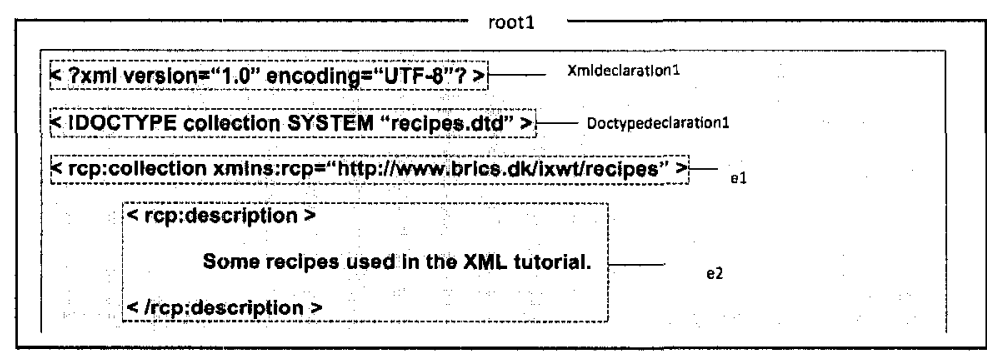

Figure 3.5: Example xml document

$$
\begin{aligned}
& \left((\text { String }: \text { name }), v_{\text {name }}\right), \\
& \left((\text { Integer }: \text { size }), v_{\text {size }}\right), \\
& \left((\text { Date }: \text { last modified time }), v_{\text {lastmodifiedtime }),}\right. \\
& \left((\text { Date }: \text { type }), v_{\text {type }}\right), \\
& \left((\text { String }: \text { extension Name }), v_{\text {extensionName }),}\right. \\
& \left((\text { String }: \text { path }), v_{\text {path }}\right), \\
& \left((\text { ID: hasItem }),\left\{\pi_{1}, \ldots, \pi_{n}\right\}\right) \\
& ]
\end{aligned}
$$

For example, XML file Recipes shown in Figure 3.4 holds an XML document root node 
Root 1 shown in Figure 3.5. Information of recipes are represented as:

File recipes[

((String:name), "recipes"),

((Integer:size), 16600),

((Date:last modified time), "2008/11/10 17:52")

((String:type), "XML Document"),

((String:extensionName), "xml"),

((String:path), "C:/Users/Qian/Desktop/paper/test/Cook Book/dish"),

((ID:hasltem), \{root1\})

]

where recipes and root 1 are ids of file Recipes and the root node of the XML document Recipes.

\subsubsection{Representation of file system}

A file system is filled with files and folders. To represent file system is basically to present files, folders, relationships between them. The PIM Model is able to present above information.

We give a small example in Figure 3.6, information of which can be presented as follows:

Folder Cook Book has subfolder Dish. Information of Cook Book is listed as follows:

Folder cookbook[

((String:name), "Cook Book"),

((Integer:size), 129000),

((Date:created time), "2008/11/18 17:16"),

((String:hasPath), "C:/Users/Qian/Desktop/paper/test")

((ID:subFolder), $\{$ dish $\})$,

]

where cookbook and dish are ids of folder Cook Book and folder Dish. 


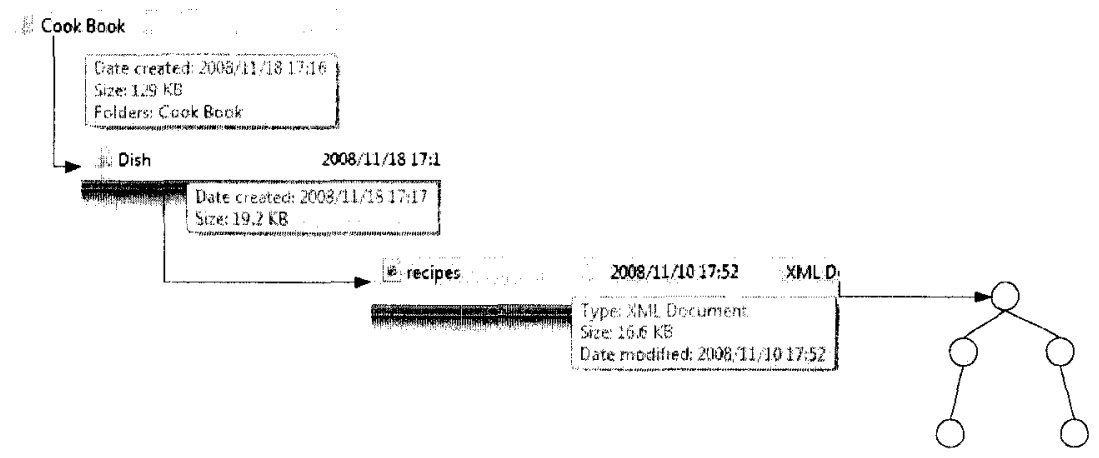

Figure 3.6: Example file system data

Folder Dish, has a file Recipes. Information related to Dish is listed as follows:

Folder dish[

((String:name), "dish"),

((Integer:size), 19200),

((ID:containsFile), \{recipes\}),

((Date:created time), "2008/11/18 17:17"),

((String:hasPath), "C:/Users/Qian/Desktop/paper/test/Cook Book")

]

where dish and recipes are ids of folder Dish and file Recipes.

File Recipes is an XML document and its root node is $X M L$. Information related to file recipes are listed as follows:

File recipes[ 
((String:name), "recipes"),

((Integer:size), 16600),

((String:type), "XML Document"),

((String:extensionName), "xml”),

((ID:hasltem), $\{$ xml $\})$

((Date:last modified time), “2008/11/10 17:52")

((String:hasPath), "C:/Users/Qian/Desktop/paper/test/Cook Book/dish")

]

where recipes and $x m l$ are ids of the XML document Recipes and the root node of the XML document Recipes.

\subsubsection{Relational Model to PIM Model}

In the relational model, a tuple is a basic unit of a relation and relations compose a relational database.

\subsubsection{Representation of a relational database}

A relational database consists of several relations, which have "ID:hasRelation" relationship with the relational database, and it has attributes: name of type String.

Assume that a relational database $\pi$ has name $v_{\text {name }}$ and it has a group of relations $\pi_{1}$, $\ldots, \pi_{n}$ for $n \geq 1$.

Relational_Database $\pi[$

((String: name), $\left.v_{\text {name }}\right)$,

((ID: hasRelation), $\left.\left\{\pi_{1}, \ldots, \pi_{n}\right\}\right)$

]

For example, as shown in Figure 3.7, database CourseSchedule, has relation Course and Teacher. Database CourseSchedule can be presented as follows:

Relational_Database courseschedule[ 


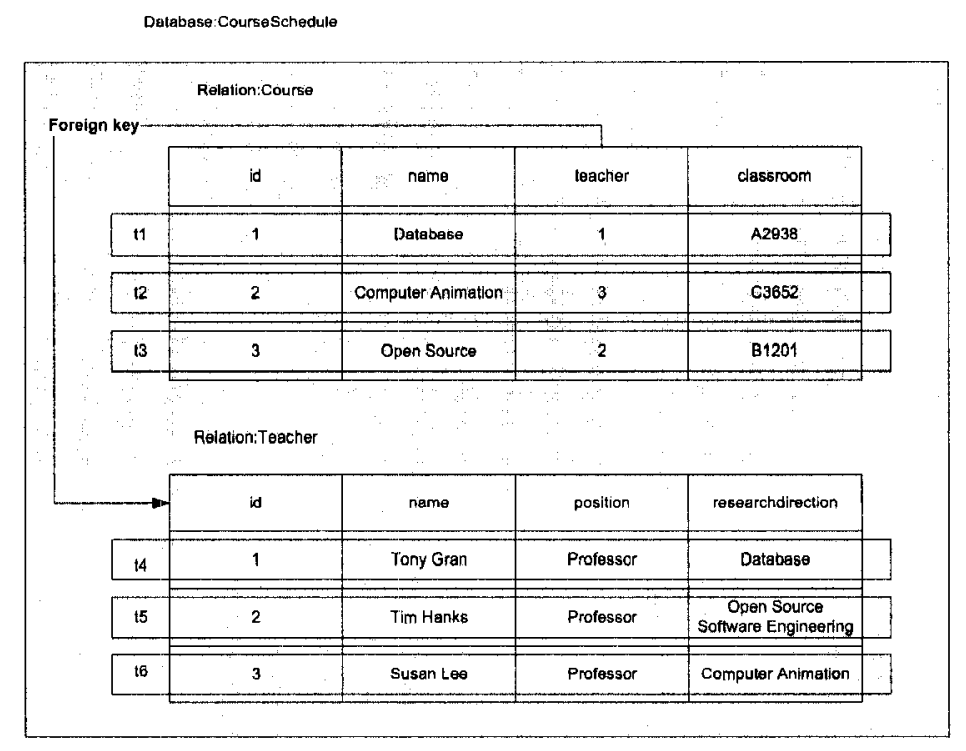

Figure 3.7: Example relational database

((ID:hasRelation), \{course, teacher\}),

((String:name), "CourseSchedule"),

]

where courseschedule is the id of the relational database CourseSchedule, course and teacher are ids of relation Course and Teacher.

\subsubsection{Representation of a relation}

A relation has attribute information and relationship information. Attribute information of a relation are: name of type String; data_length of type Integer. A relation contains tuples and it has "ID:hasTuple" relationships with tuples it contains.

Assume that relation information $\pi$ 's name is $v_{\text {name }}$, and its data length is $v_{\text {datalength }}$. Also assume that relation $\pi$ has a group of tuples $\pi_{1}, \ldots, \pi_{n}$ for $n \geq 1$. Information of $\pi$ has the following form: 
Relation $\pi[$

$$
\begin{aligned}
& \left((\text { String : name }), v_{\text {name }}\right), \\
& \left((\text { String : datalength }), v_{\text {datalength }}\right), \\
& \left((\text { ID: hasTuple }),\left\{\pi_{1}, \ldots, \pi_{n}\right\}\right) \\
& \text { ] }
\end{aligned}
$$

For example, relation Course's "data length" is $4096 \mathrm{~KB}$. It also has tuple t1, tuple t2, tuple $\mathrm{t} 3$. Information of the relation Course can be represented as follows:

Relation course[

((String:name), "course")

((Integer:data length), 4096000)

((ID:hasTuple), $\{\mathrm{t} 1, \mathrm{t} 2, \mathrm{t} 3\})$

]

where course, $t 1, t 2$ and $t 3$ are ids of relation Course, tuple $T 1, T 2$ and $T 3$.

\subsubsection{Representation of a relational tuple}

A tuple has a group of attributes. Some attributes are foreign keys to represent relationships between relations. In the PIM Model, we use information item id to represent tuple so we need to convert foreign key references to id references.

Assume that a relation tuple has non-foreign key attributes $a_{1}, \ldots a_{n}$ of types $t_{1}, \ldots t_{n}$ with values $v_{1}, \ldots v_{n}$ for $n \geq 1$ and foreign keys $k_{1}, \ldots, k_{m}$ referring to tuple $\pi_{1}, \ldots, \pi_{m}$ for $m \geq 0$. The rule for presenting information of a tuple has the following form:

Tuple $\pi[$ 


$$
\begin{aligned}
& \left(\left(t_{1}: a_{1}\right), v_{1}\right), \\
& \ldots, \\
& \left(\left(t_{n}: a_{n}\right), v_{n}\right), \\
& \left(\left(I D: k_{1}\right), \pi_{1}\right), \\
& \ldots, \\
& \left(\left(I D: k_{m}\right), \pi_{m}\right), \\
& ]
\end{aligned}
$$

For example, as shown in Figure 3.7, there is a relation Course, in which there are four attributes "id", "name", "teacher" and "classroom", among which, id's is of Integer, name is of String, teacher refers to id of a tuple in table teacher, and classroom is of String. In relation Course, there is a tuple $t 1$. We can present information of tuple $t I$ as follows:

Tuple $\mathrm{t} 1[$

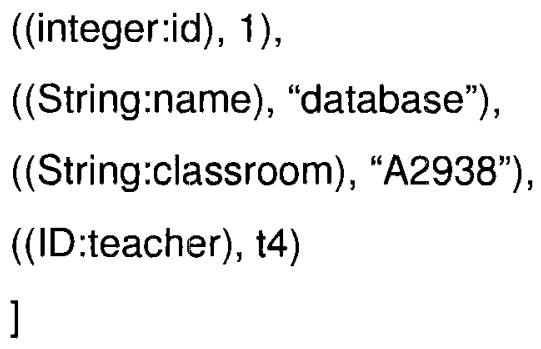

\subsubsection{XML Document to PIM Model}

An XML document consists of a root node, which represents the whole XML tree, processing introduction nodes, element nodes, text nodes, comment nodes, document node and attribute nodes.

\subsubsection{Representation of a root node}

Each XML document has one root node which represents the whole XML tree. Root node has children node: processing introduction nodes, document node, $\mathrm{xml}$ element nodes and 
comment nodes.

Assume that an XML root node $\pi$ has the following information: a group of processing introduction nodes $p_{1}, \ldots, p_{n}$ for $n \geq 0$; an XML element $\pi_{1}$; a document node D; comment, whose data type is String and whose value is $v_{\text {comment }}$. Information of $\pi$ can be represented as follows:

$$
\begin{aligned}
\text { Root } \pi[ & \\
& \left((I D: \text { hasProcessing }),\left\{p_{1}, \ldots p_{n}\right\}\right), \\
& \left((I D: \text { hasXMLElement }), \pi_{1}\right) \\
& ((\text { ID : hasDocument }), D) \\
& \left((\text { String }: \text { comment }), v_{\text {comment }}\right)
\end{aligned}
$$

For the XML document shown in Figure 3.5, information of root is represented as:

Root root[

$$
\begin{aligned}
& \text { ((ID:hasProcessing), }\{\text { xmldeclaration } 1\}), \\
& \text { ((ID:hasXMLElement), }\{\mathrm{e} 1\}), \\
& \text { ((ID:hasDocument), doctypedeclaration1) } \\
& \text { ] }
\end{aligned}
$$

\subsubsection{Representation of an XML element}

An XML element has information: name of data type is String; attributes of data type String; content of data type String; children XML elements;

Assume that there is an XML element $\pi$, its name is $v_{\text {name }}$, it might have a group of attributes $a_{1}, \ldots, a_{n}$ with values $v_{1}, \ldots, v_{n}$ for $n \geq 0$, it might have a group of children XML elements $\pi_{1}, \ldots \pi_{m}$ for $m \geq 0$ and it might have content with value $v_{\text {content }}$. Information of $\pi$ can be represented as follows:

$X M L$ Element $\pi[$ 


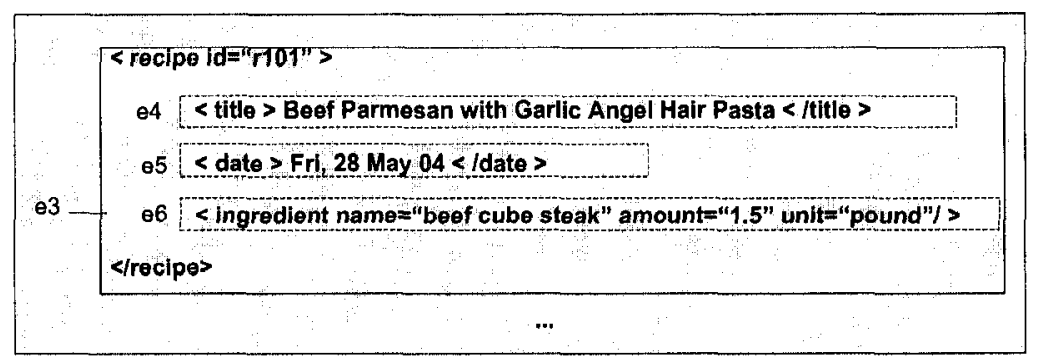

Figure 3.8: Example XML elements

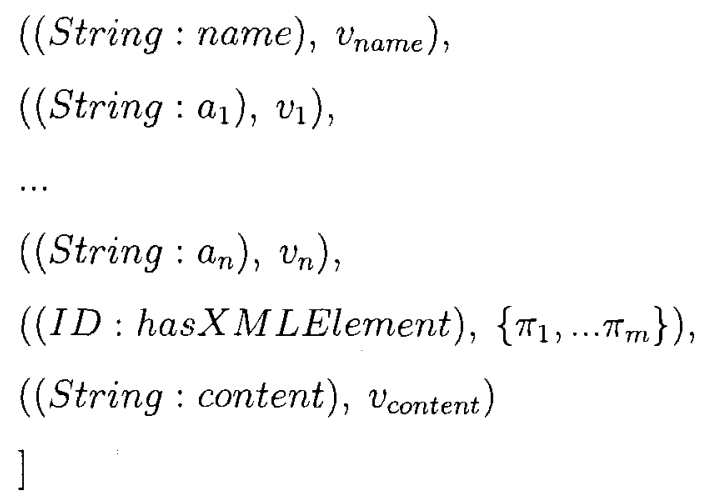

For example XML element $e 3$ shown in Figure 3.8 has the following form:

\section{XMLElement $e 3$ [}

((String:name), "recipe"),

((String:id), "101"),

((ID:hasXMLElement), $\{e 4, e 5, e 6\})$,

\section{]}

\subsubsection{Representation of processing introduction}

An XML declaration has version information with value String and it has encoding information with value String. 


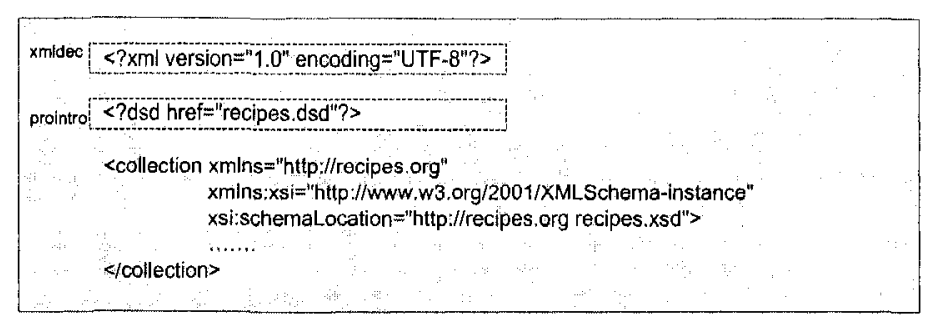

Figure 3.9: Example processing instruction nodes

Assume that there is an XML declaration node $\pi$. It shows the version of the XML document of $v_{\text {version }}$ and the encoding with value $v_{\text {encoding. }}$. Information of an XML declaration node has the following form:

XML_Declaration $\pi[$

$$
\begin{aligned}
& \left((\text { String }: \text { version }), v_{\text {version }}\right), \\
& \left((\text { String }: \text { encoding }), v_{\text {encoding }}\right), \\
& \text { ] }
\end{aligned}
$$

Processing introduction other than XML declaration node refers to the file assigned by attribute "href".

Assume that there is a processing introduction node $\pi$. The value of its attribute "href" refers to a processing introduction file $\pi_{1}$.

$$
\begin{gathered}
\text { Processing_Introduction } \pi[ \\
\left((I D: \operatorname{refer} T o), \pi_{1}\right), \\
]
\end{gathered}
$$

For example for processing introductions shown in Figure 3.9, xmldeclaration and processingintroduction are represented as:

XML_declaration xmldec[ 


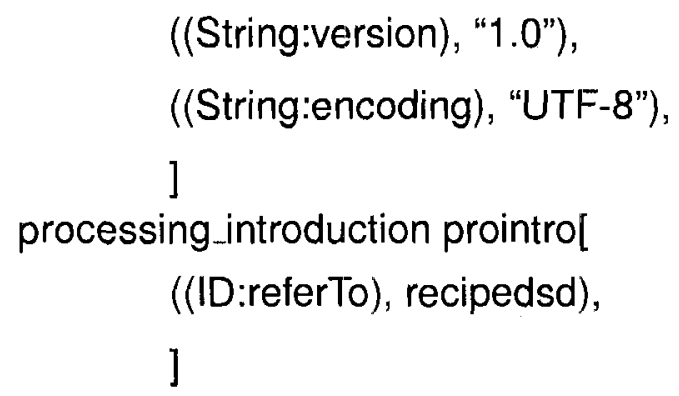

A document node is a special node which indicates where to find the DTD file for the XML document (note that we only consider the situation in which DTD is outside the XML document)

Assume that there is a document node $\pi$ and it indicates a Uniform Resource Locator (URL), which indicates where to find the DTD file $\pi_{1}$. We define that $\pi$ has relationship "ID:referTo" to $\pi_{1}$. The information of $\pi$ can be represented as the following form:

Doctype_Declaration $\pi[$

$\left((I D: \operatorname{refer} T o), \pi_{1}\right)$

]

The doctype declaration shown in Figure 3.5 is presented as:

Doctype_declaration doctypedeclaration1[

((ID:referTo), recipesdtd)

]

\subsubsection{Emails to PIM Model}

We simply define three classes in emails: message, attachment and person. A message refers to an email message. An attachment refers to an attachment attached in the message. A person refers to a sender who sends an email to the user.

A message has information including subject, content, received date, attachment and sender information and their types are String, String, Date, ID and ID. Assume that an email message $\pi$ has subject $v_{\text {suject }}$, content $v_{\text {content }}$, received_date $v_{\text {received_date, attach- }}$ ments $a_{1}, \ldots a_{n}$ for $n \geq 0$ and it has sender $\pi_{1}$. An email message can be presented as the 
following form:

$$
\begin{aligned}
& \text { Message } \pi[ \\
&\left((\text { String }: \text { subject }), v_{\text {subject }}\right), \\
&\left((\text { String }: \text { content }), v_{\text {content }}\right), \\
&\left((\text { Date }: \text { receiveddate }), v_{\text {receiveddate }}\right), \\
&\left((I D: \text { attachment }),\left\{a_{1}, \ldots a_{n}\right\}\right) \\
&\left((I D: \text { sender }), \pi_{1}\right)
\end{aligned}
$$

Information of a sender includes: sender's name of data type String and his email address of data type String. Assume that a sender $\pi$ 's name is $v_{\text {name }}$ and his email address is $v_{\text {emailAddress }}$, the rule to present information for a sender has the following form:

\section{Sender $\pi[$}

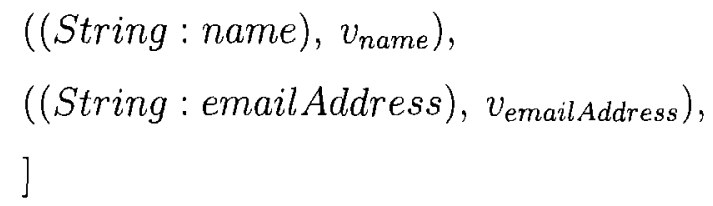

Information of an attachment includes: name of data type String, size of data type Integer, extension name of data type String and content of data type String. Assume that an attachment $\pi$ 's name is $v_{\text {name }}$, its size is $v_{\text {size }}$, its extension name is $v_{\text {extensionName }}$ and its content is $v_{\text {content }}$. The rule to present information for an attachment has the following form:

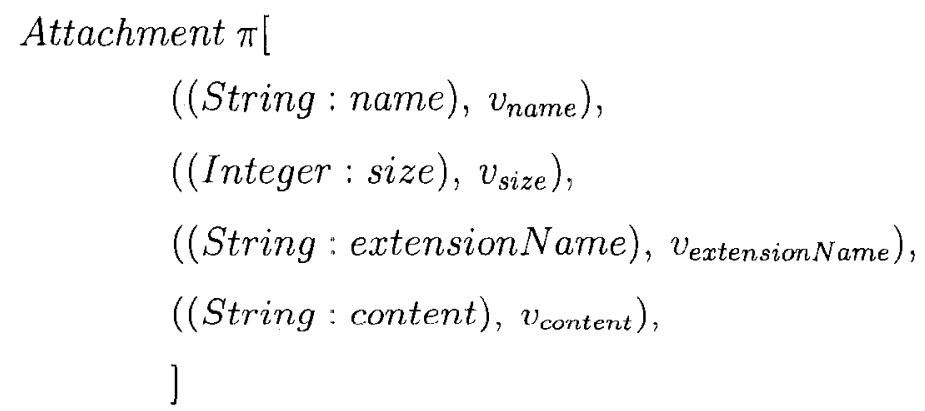

For example, there is an email message shown in Figure 3.10, its information can be presented as follows: 


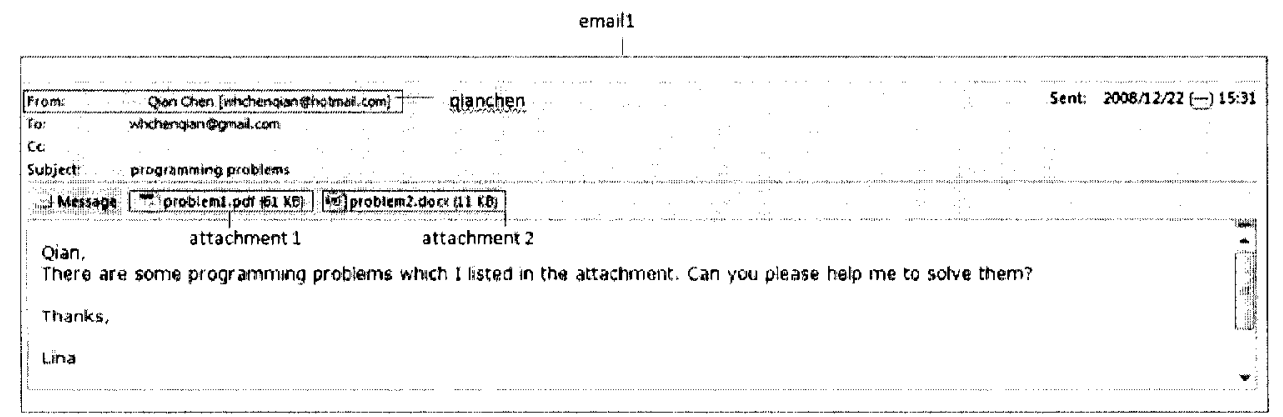

Figure 3.10: Example email message

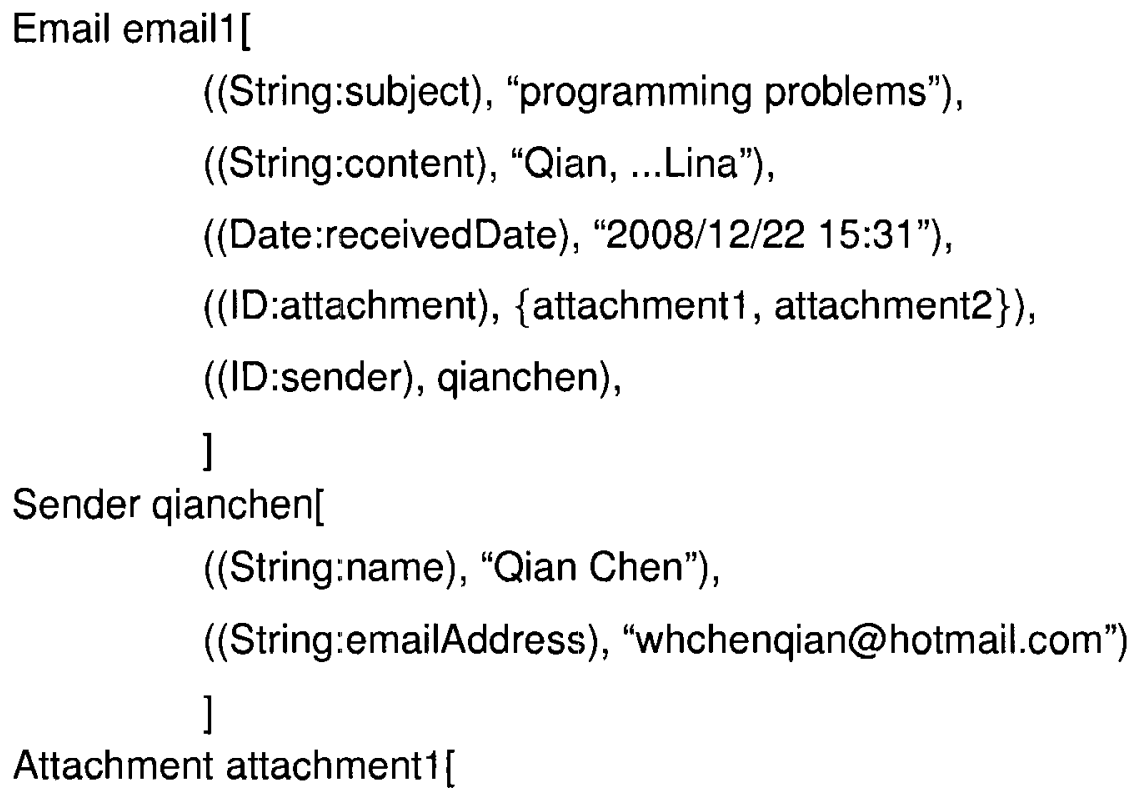




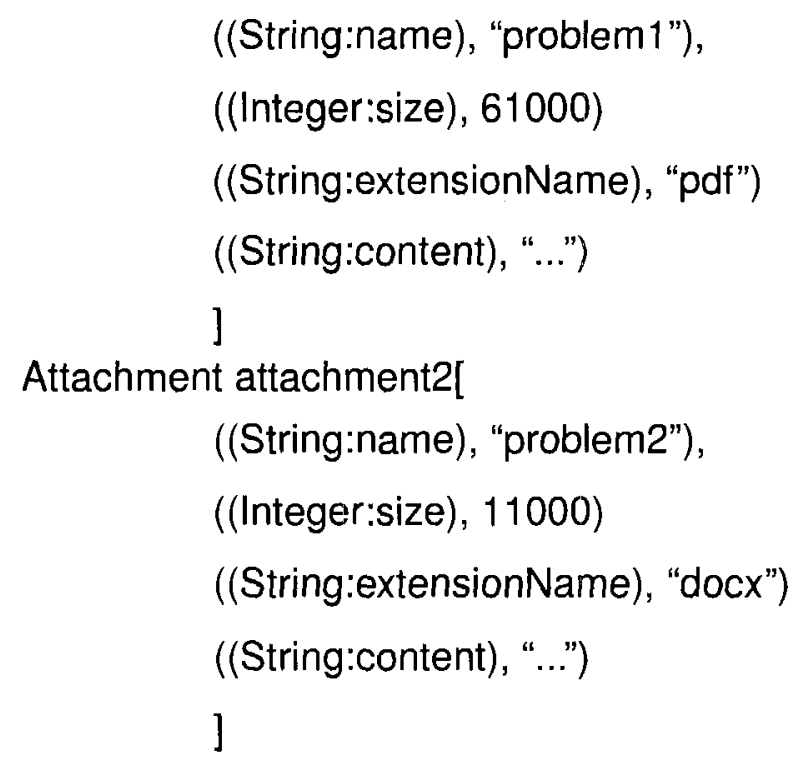

\subsection{Formation of a graph view}

Data residing at heterogeneous data sources can be represented as information items with the the PIM Model. Each information item is identified by an instance of id. Attribute names or relationship names are properties. Values are attribute values or information items which are related to the information item. Because all data are represented uniformly in the PIM Model and information items are connected through their ids, heterogeneous data are eventually represented as a uniform graph view.

We use a simple example to show how to form a graph view for example data illustrated in Figure 3.11. The example data consists of folders and files, among which there is an XML document. The content of the XML document is partially displayed. We represent these data in a graph view as shown in Figure 3.12. Note that information item $I_{4}$ has has Item relationship with information item $I_{6}, I_{6}$ is root element of the XML document and $I_{7}, I_{8}, I_{9}$ are information items inside $I_{6}$. Both of information item $I_{3}$ and information item $I_{7}$ connect to information item $I_{5} . I_{5}$ is connected to information item in file system and information item inside XML document at the same time, which forms a graph. 


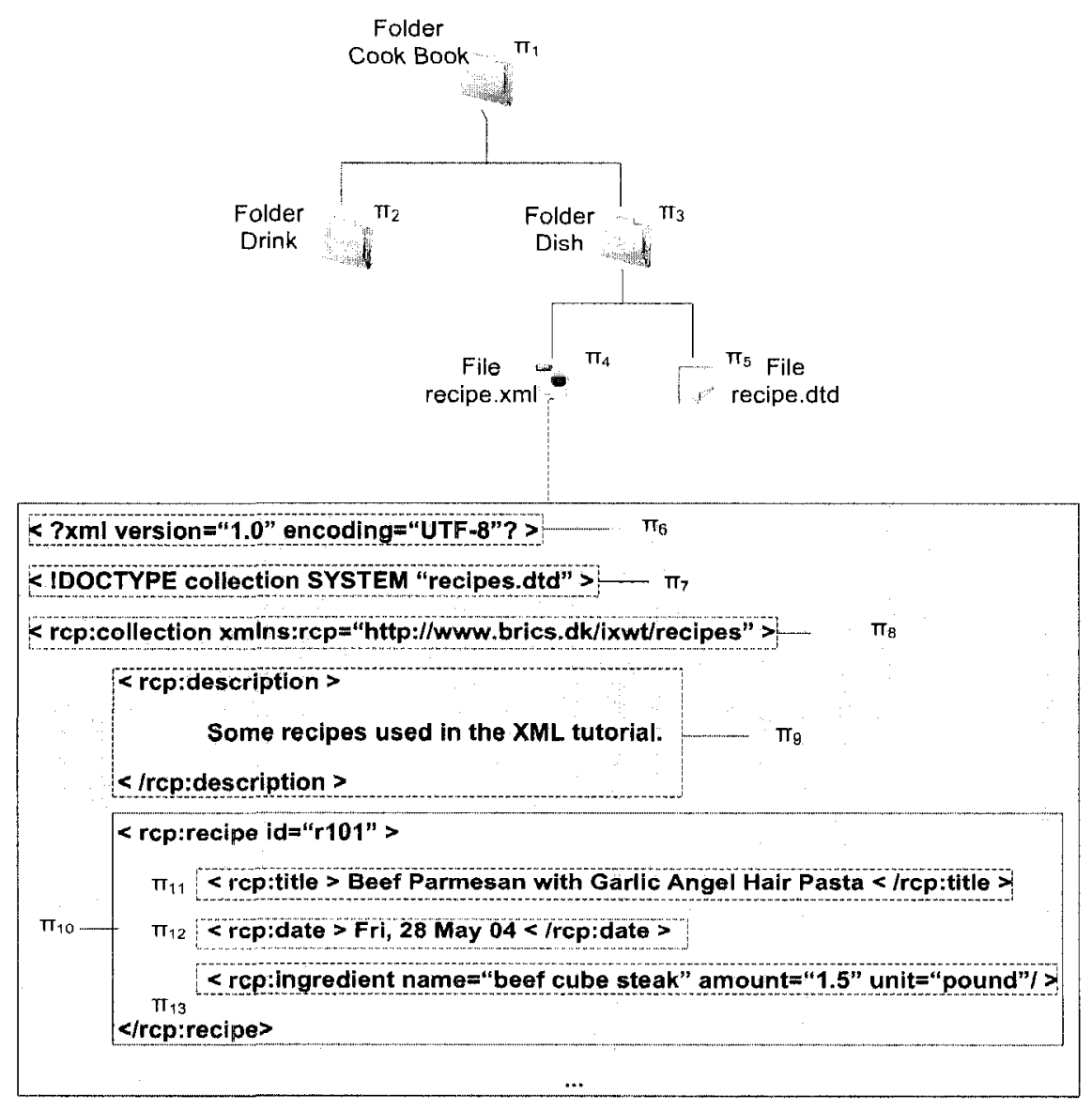

Figure 3.11: Example data 


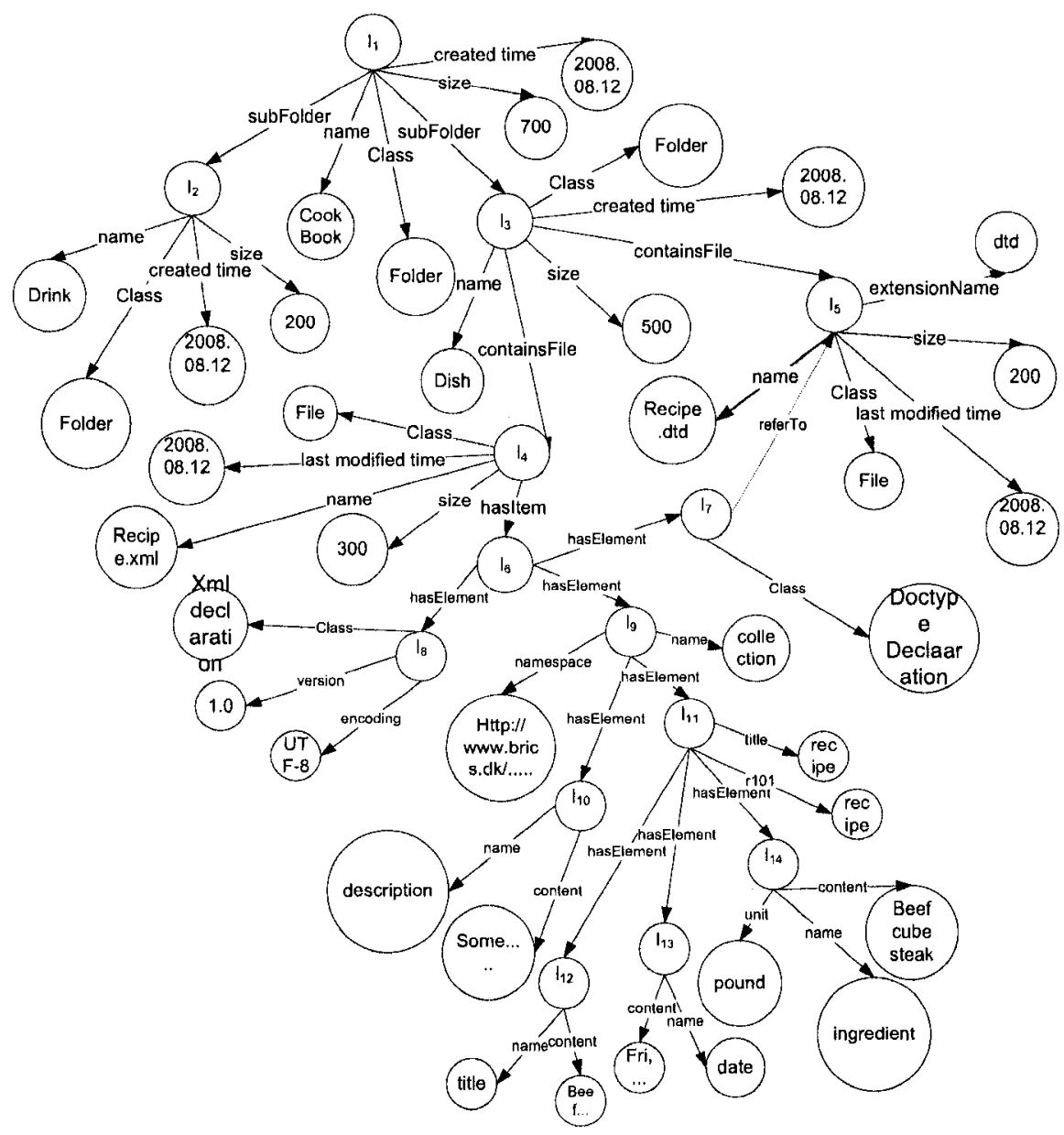

Figure 3.12: Example graph view 


\subsection{Features of the PIM Model}

We summarize the features of the PIM Model as follows:

- Data in heterogeneous data sources have different formats and diverse data types and it is difficult to represent them in a single table. Our PIM Model makes representation of such data easy by reducing the effort of predefining the schema. Heterogeneous data are converted to information items. Through their ids, information items can be connected to form a uniform view.

- Information items can belong to multiple classes and classes can be organized hierarchically.

- Dataspace requires representation of heterogeneous data in a uniform way, requires data to be integrated loosely, requires to provide services upon coexistent data and allows coexistent data to be integrated further. Our PIM Model represents heterogeneous data in a uniform graph view. Over the view, structured query and keyword search services are able to provided. The view has simple format and it allows semantic integration upon it. Therefore, it fulfills the Dataspace vision. 


\section{Chapter 4}

\section{Implementation}

In this chapter, we describe in detail the design and implementation of our Personal Information Management System that is based on the PIM Model. Our system is designed to manage personal information on a user's computer. This task is accomplished through three layers: the physical layer stores information items in a storage; the logical layer consists of operations conducted over the storage; the interface layer, which allows users to send requests to and receive results from the logical layer.

\subsection{System overview}

We have implemented our Personal Information Management System as a web application in Google Web Toolkit (GWT) [11] and have stored information items in a Berkeley DB [12],

Google Web Toolkit Considering the extensibility of our Personal Information Management System, we decide to develop our system as a web application so that it can be used remotely after further development. It is currently running on Localhost. When developing 
a web application, building, reusing and maintaining large JavaScript code and AJAX components is always difficult and fragile. Though there are many open source frameworks available for building web applications, few of them can ease this burden. Google Web Toolkit (GWT) boasts significant community support and successfully reduces this burden. GWT allows developers to quickly build and maintain complex, yet efficient, JavaScript front-end applications in the Java programming language [11]. It has many features that promote users to develop web applications: it communicates with the server through simple Remote Producer Calls (RPC); it reuses User Interface (UI) components across projects by creating reusable widgets; and it allows developers to write AJAX in the Java language and compile the Java code into optimized JavaScript. Based on GWT's develop-friendly features, we decide to develop our web application in their provided framework.

Berkeley DB There are many options to store data in our PIM system, such as storing our PIM Model in XML databases like Apache XIndice [7], RDF databases like Jena [8], relational databases like MySql [9] and object-relational databases like Oracle [10]. One of our system goals is to develop a structured query language for our PIM system. Our requirements include having a simple and efficient database without supporting any query language. Hence, a DBMS is not necessary in our system. After considering our stipulations, we have chosen the Berkeley Database (Berkeley DB), which is a family of open source embeddable databases. It allows developers to incorporate into their applications a fast, scalable and transactional database engine with industrial grade reliability and availability. Berkeley DB eliminates the overhead of SQL and interposes communication; it is completely embedded within the application and invisible to end-users. Because it is embedded, the application lowers implementation, licensing and hardware costs, as well as ongoing administration costs. It supports high flexibility and control, which allows developers to configure many Berkeley DB's aspects and optimize it for specific applications. It stores arbitrary key/value pairs as byte arrays, and supports multiple data items for a single 
key.

Physical Layer It contains the storage which stores all information items that are converted from the personal information on a user's computer. We apply Berkeley DB to store those information items.

Logical Layer It consists of operations conducted over the storage. It mainly contains data collection and query.

Interfaces It includes user login, data collection, browse, search and customization. Users send requests to the logical layer and view the subsequent returned results .

The system architecture is briefly described in Figure 4.1. We will discuss in detail the storage, data extraction and queries in the following sections.

\subsection{Storage}

The information items are stored in a Berkeley DB. There are four primary databases: property database, entities database, class database and subClass database. For the property database, we build a property index file. For the entities database, we build one propertyobject index file for each property. The schema of the databases and index files are shown in Figure 4.2.

Property Some properties might appear repeatedly. In order to save space in the storage, we build a property database to store all properties. The database generates an identification for each property and then stores the type and name of the property in a string, which has the format type:property. 


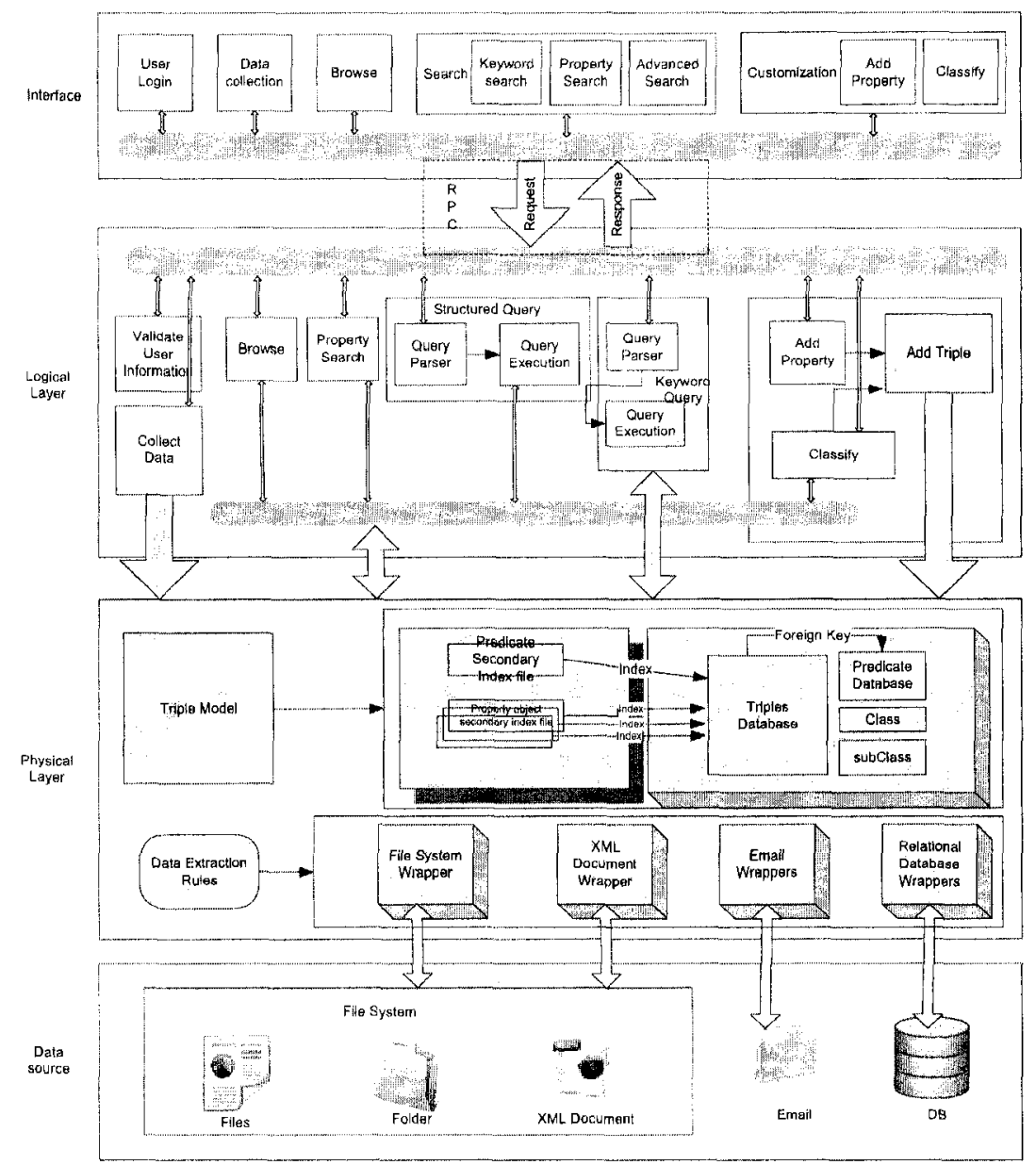

Figure 4.1: System Architecture 


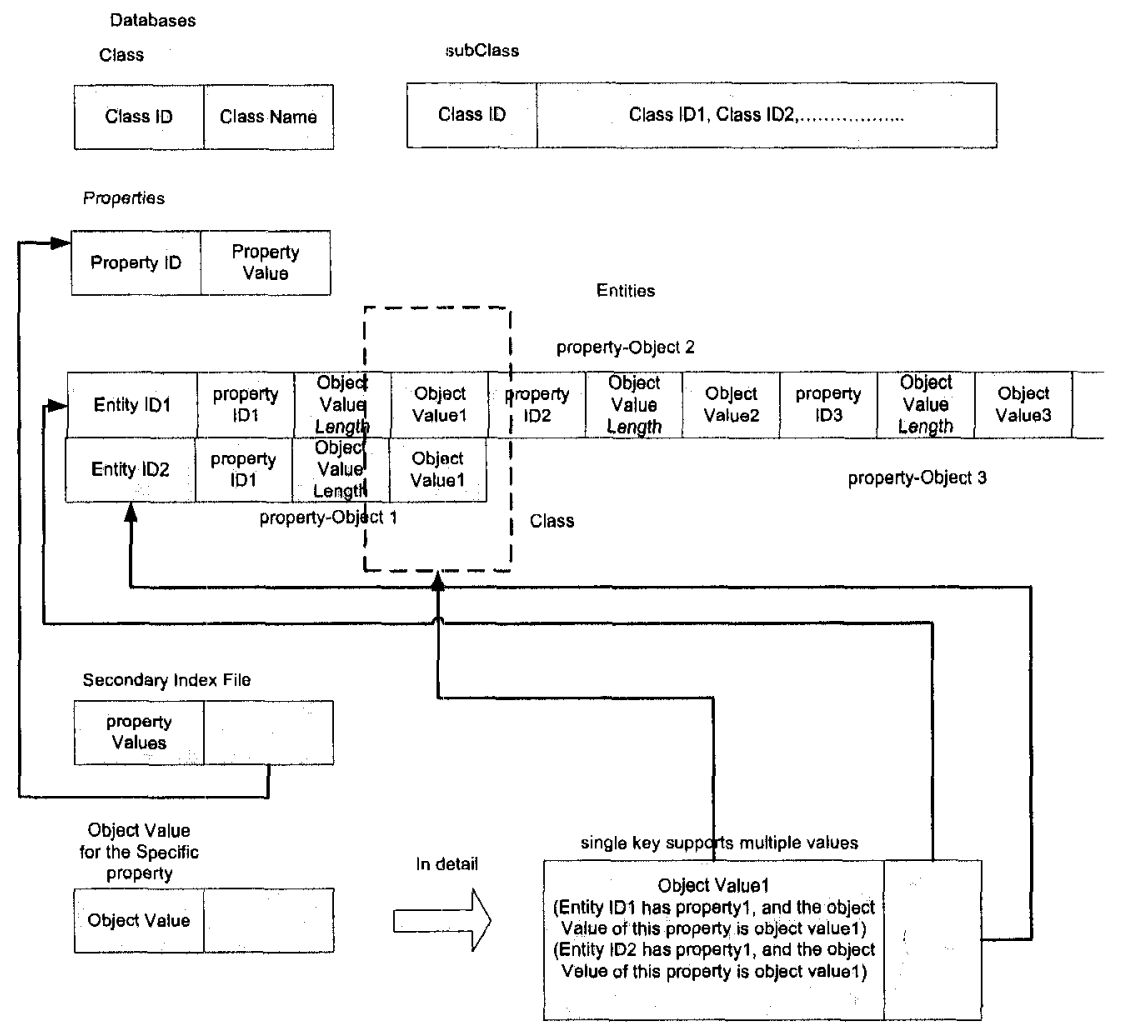

Figure 4.2: The schema of the databases 
Entities This database stores information items. As Berkeley DB actually stores sets of key/value pairs, there are only two columns in entities database, which comprise entity id and a string, which contains all of the entities' properties and their values. The value column consists of a set of property-object units. Each property-object unit contains three parts: property-id, object value length and the object value, where, the type of property-id is integer, the type of object value length is integer and the type of the object value is String. Note that these three parts are sequentially stored. In this way, all attributes information of an entity and the relationships between the entity and other entities are all stored in one row of the database.

Class This database stores classes. It generates an identification for each class. In Figure 4.3, we define classes Folder, File, Cook, Food, Beverage, Recipe, Food Recipe and Beverage Recipe.

SubClass This database stores subClasses for classes. There are two columns: key and value. Key column stores class id and value column stores ids of subClasses of the corresponding class. Value column comprise subClasses ids which are separated by commas. In Figure 4.3, class Cook has subclasses Food and Beverage. Recipe has subclasses Food Recipe and Beverage Recipe. Information item Cook Book belongs to classes Folder and Cook. Information item Drink belongs to classes Folder and Beverage. Information item Dish belongs to classes Folder and Food. Information items Recipe.xml and Recipe.dtd belong to classes File and Food Recipe.

Property Index File This index file is essentially an index for the property database. It is designed for the occasion where the property name is known and we want to search for the property id. 
Property-Object Index Files These index files are actually indexes for the entities database. For each property, there is a property-object index file which is named according to the property id. A property-object index file stores corresponding objects of the property as keys. The key points to the records in the entities database. Those records contain values of those keys as objects. Example data in Figure 3.11 is stored in the storage as shown in Figure 4.3. In Figure 4.3, the id of the property String:Class is 1 . An index file called 1 is built and it is the index of all object values for the property String:Class . In this example, object values of the property String:Class include "Folder", "File", "Cook", "Food", "Beverage", "Recipe", "Food Recipe", "Beverage Recipe", which are keys. "Folder" points to records which contain "Folder" as an object in the entities database. Therefore, "Folder" points to three records whose ids are 1, 2 and 3 in the entities database. "File" points to records which contain "File" as object in the entities database. Therefore, "File" points to two records, whose ids are 4 and 5 in the entities database. Note that a single key in an index file can support multiple data items.

\subsection{Data Collection}

This section demonstrates how to extract data from heterogeneous data sources and then insert it into storage.

\subsubsection{Data Extraction}

One of significant approaches for extracting data from information sources is to write specialized programs, generally called wrappers. These programs extract certain pieces of information from structured or semi-structured data into a uniform format, such as XML.

Though heterogeneous information has various presentative formats as their interfaces, they usually employ a few common data models as underlying databases. Most of personal 
Class

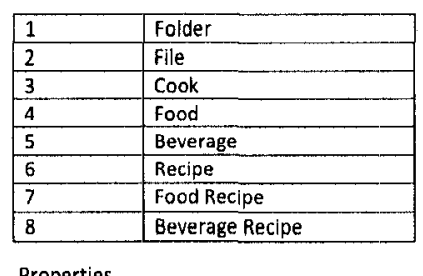

Properties

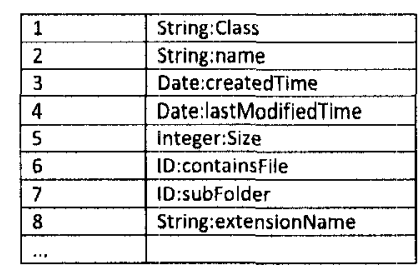

Entities
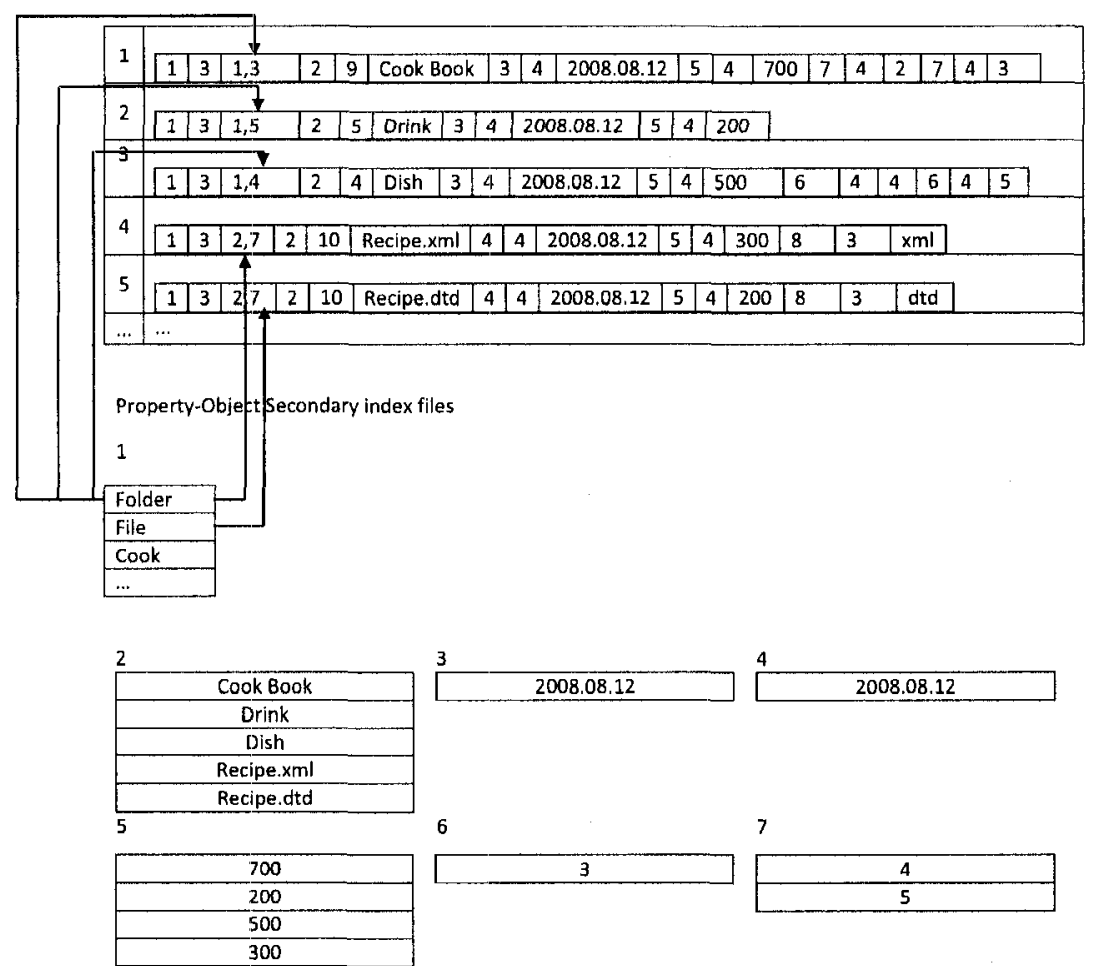

8

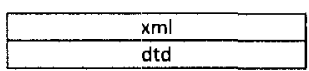

Figure 4.3: Store data in databases 
information allows us to directly access their underlying database. Therefore, it is only required that we develop core wrappers for the common data models and then only configure them for accessing information sources.

For example, we now need to extract data from MySQL databases and Microsoft SQL Server databases. As they are two relational databases, they have common data model. We can therefore develop one wrapper for extracting data from both of them. We also need to configure the core wrapper to access each of them. Another example is to extract data from email servers and clients. Regardless from which email server we extract information, only one core wrapper is required for extracting, because email massages share the same format.

Chapter 3 discussed ways to present various data models with the PIM Model. We extract data from such various data model following and convert them into information items. We have developed wrappers for file system, relational databases, XML documents and email clients.

As shown in Figure 4.4, for extracting data from a file system, we use Java programming to retrieve attribute information and extract relationship information of folders and files. Data is extracted from relational databases by using JDBC to connect such databases, using SQL to retrieve the stored data and then using algorithms to attain relationships between the databases, relations and tuples. In order to extract data from XML documents, the Simple API for XML (SAX) to parse XML documents is used to parse XML documents. For extracting email information, JAVAMail API is used to connect to email servers and then retrieve data from them. Personal information is extracted through the use of wrappers and is converted into pieces of information items.

\subsubsection{Data Loading}

Information items are stored in the entities database. How an information item is added into the storage are as follows: 


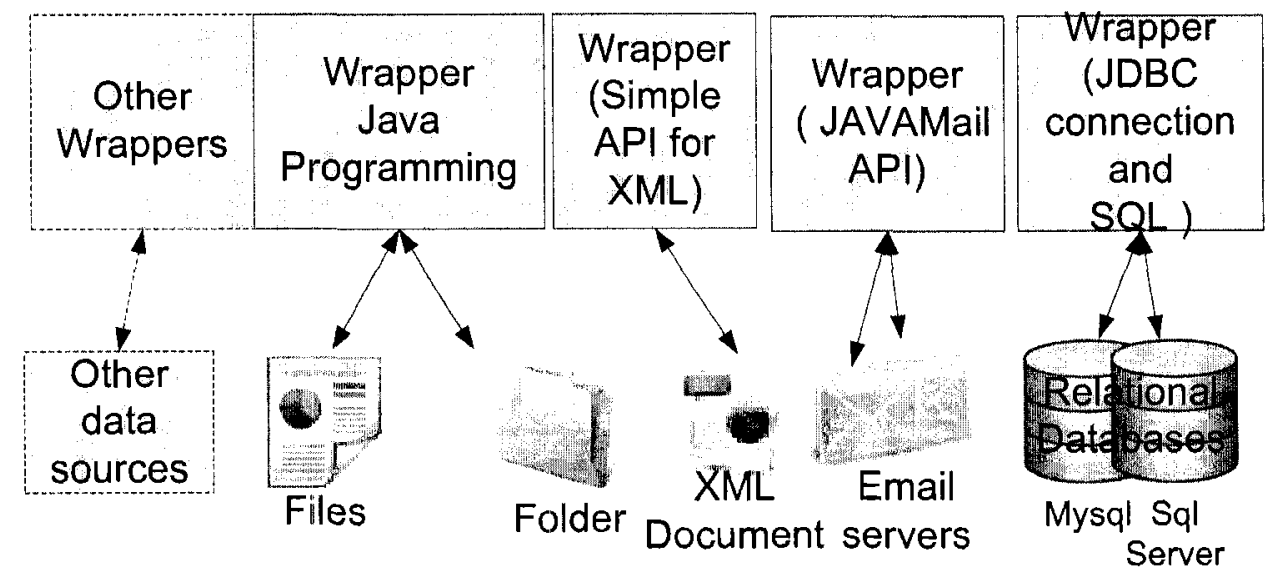

Figure 4.4: Wrappers

- Id of entities database increments.

- The property is checked in property database. If the property is in the property database, the property id is returned; otherwise the property id increments, the property is added into the property database and the maxim id in the property database is returned. If the property is String:Class, the value of the property is to be checked in class database. If the value is in the class database, the class id is returned; otherwise the class id increments, the value is added into the class database. Class id is assigned to the property value,

- The set of the (property id, object value length, object) list of the information item is retrieved from entities database. If the property id and the object to be added have already been in the (property id, object value length, object) list, return false; Otherwise, the (property id, object value length, object) list is appended into the 


\begin{tabular}{|c|c|}
\hline Alg & ithm: getPropertylD \\
\hline $\ln p$ & property \\
\hline Ou & ut: ID of the property \\
\hline 1 & initialize preid to zero \\
\hline 2 & IF property is in property index file then \\
\hline 3 & Set pried to propertyid of the property \\
\hline 4 & ELSE \\
\hline 5 & add property to property index file \\
\hline 6 & maxpropertyID increments \\
\hline 7 & Set pried to maxpropertyID \\
\hline 8 & END IF \\
\hline 9 & Return preid \\
\hline
\end{tabular}

Figure 4.5: Algorithm of inserting a property

(property id, object value length, object) to a new list. The new (property id, object value length, object) list replaces the old one and true is returned.

The information item addition consists of three main functions: addProperty, addSubject and addPropertyObject. The algorithms are shown in Figure 4.5, Figure 4.6 and Figure 4.7.

\subsection{Query}

Our system supports two types of queries: structured queries and keyword searches. The following subsections include detailed discussions of both types of queries. 


\begin{tabular}{|c|c|}
\hline \multicolumn{2}{|c|}{ Algorithm: addSubject } \\
\hline \multicolumn{2}{|c|}{ Input: Property } \\
\hline \multicolumn{2}{|c|}{ Output: ID of the entity } \\
\hline 1 & Initialize subID to zero \\
\hline 2 & Initialize maxID to zero \\
\hline 3 & Call getPropertyID with Property returning preID \\
\hline 4 & Set maxID to maxEntityID of the Entities index file \\
\hline 5 & maxID increments \\
\hline 6 & Set maxEntityID to maxID \\
\hline 7 & add maxID, preID, Property to Entities index file \\
\hline 8 & return maxID \\
\hline
\end{tabular}

Figure 4.6: Algorithm of inserting an information item

\subsubsection{Structured Query}

The structured query is similar to SQL. Our structured query has a statement for retrieving information from the storage: the select statement. The select statement, is also a mapping or a select-from-where block, is formed of the three clauses select, from, and where and has the following form:

\section{SELECT variable list}

FROM information item pattern expression

WHERE filter condition

where variable list is a list of variables whose values are to be retrieved by the query for $1 \leq i \leq n$; Information item pattern expression indicates what pattern the information items should match. Filter condition is a conditional expression that contains any expression evaluating to a boolean value and eliminates information items that do not satisfy the 


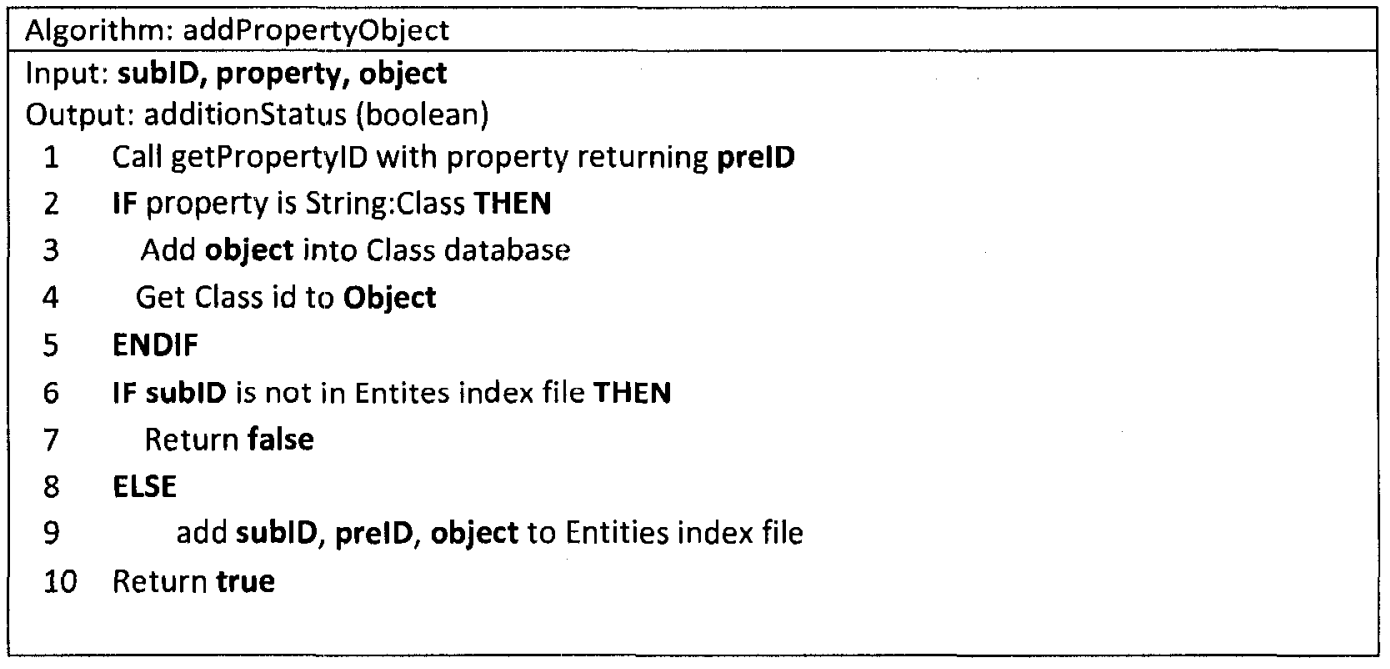

Figure 4.7: Algorithm of inserting a property/object pair

condition.

The information item pattern expression consists of a set of information item path expressions. The information item path expression has the following declaration:

$$
c_{1}, \ldots c_{m} \circ\left[\left(p_{1}, v_{1}\right), \ldots,\left(p_{n}, v_{n}\right)\right]
$$

where $o$ is a variable or a value and it matches the id of an information item; $c_{j} \in C$ is a variable or a value and it matches an information item class of $o$ for $0 \leq j \leq m$; $p_{i}$ can be a single value or a variable for $1 \leq i \leq n$ and it matches a property of $o$; $v_{i}$ can be a single value or a set of values and can also be a single variable, or a set of variables for $1 \leq i \leq n$ and it matches the value of $p_{i}$. Note that, $c_{1}, \ldots c_{n}$ and $\left(p_{1}, v_{1}\right), \ldots,\left(p_{n}, v_{n}\right)$ are optional.

The following information item path expression illustrates an information item that belongs to classes Folder and Cook: 


\section{Folder, Cook $\$ \times[]$}

where $\$ \mathrm{x}$ is a variable matching the id of the information item that belongs to class Folder and Cook.

The following information item path expression illustrates an information item that has a property name and a property containsFile:

Folder \$x [(name, \$y), (containsFile, \$z)]

where $\$ \mathrm{x}$ is a variable matching the id of the information item that belongs to class Folder and has a property name and a property containsFile; $\$ y$ is a variable matching the value of the property name; $\$ \mathrm{z}$ is a variable matching the value of the property containsFile.

The following information item path expression illustrates an information item that has the property size with value 1000, has a property path and belongs to classes File and Food:

File, Food \$z [(size, 1000), (path, \$p)]

where $\$ \mathrm{z}$ is a variable matching the id of the information item which belongs to classes File and Food and has the property size with value 1000 and a property path; $\$ \mathrm{p}$ is a variable matching the value of the property path.

Consider the following information item path expression:

File, Paper \$q [(name, paper), (size, 500)]

where $\$ \mathrm{q}$ is a variable matching the id of the information item that belongs to classes File and Paper, has property name with value paper and has the property size with value 500 .

The information item pattern expression contains a set of information item path expressions. The following information item pattern expression illustrates that an information item $\$ \mathrm{x}$ belongs to class Folder and it contains two kinds of files $\$ \mathrm{z}$ and $\$ \mathrm{q}$. $\$ \mathrm{z}$ belongs to classes File and Food, has the size property with value 10000 and has the path property with value \$w. \$q belongs to classes File and Paper, has the name property with value paper and has the size property with value 500:

Folder $\$ x[($ name, $\$ y)$, (containsFile, $\{\$ z, \$ q\})]$;

File, Food $\$ z[($ size, 10000), (path, \$w)]; 
File, Paper \$q [(name, paper), (size, 500)]

Next we give some examples for form (4.1):

(1) select $\$ y$

from \$y \$x[(name, Cook Book)]

(2) select $\$ z$

from Folder, Cook $\$ x[($ subFolder, $\$ y)$, (name, Cook Book)];

Folder, Food \$y[(name, \$z)]

(3) select $\$ p$

from Folder, \$y $\$ x[($ name, Cook Book $)]$;

$\$ y \$ m[($ name, $\$ p)]$

(4) select $\$ n$

from Folder $\$ \times[($ name, $\$ n)$, (containsFile, $\$ y)]$;

File, Recipe $\$ y[($ name, $\$ z)$, (size, \$p)]

where $\$ p>1000$ or $\$ z=$ paper

The first query asks for the class name $\$ y$ for the information item $\$ \mathrm{x}$ called Cook Book. The second query asks for the name $\$ z$ of the information item $\$ y$ which belongs to classes Folder and Food; $\$ y$ is also a subfolder of the information item \$x called Cook Book which belongs to classes Folder and Cook. The third query asks for the name $\$ p$ of the information item $\$ \mathrm{~m}$, which belongs to class $\$ \mathrm{y}$ other than class Folder and $\$ \mathrm{y}$ is also a class of the information item $\$ \mathrm{x}$ called Cook Book. The fourth query asks for the name $\$ \mathrm{n}$ of Folder information item $\$ \mathrm{x}$ that contains file information items $\$ \mathrm{y}$, which also belong to class Recipe; whose name $\$ \mathrm{z}$ is called paper, or whose size $\$ \mathrm{p}$ is larger than 1000. 


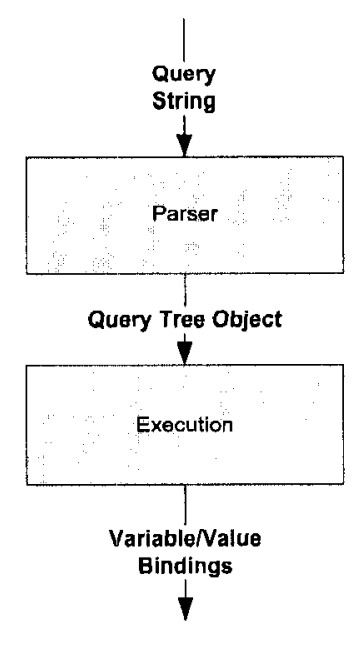

Figure 4.8: Query Processing

\subsubsection{Structured Query Processing}

To process a query, there are two main components: parser and execution. The processing is illustrated in Figure 4.8.

The parser consists of a tree, and actions inserted in each node of the tree. The tree is actually jjtree, which is generated by javacc. Javacc is an open source parser generator for the Java programming language. It generates a parser tree for a formal grammar provided in EBNF notation. In each node of the tree, actions are inserted. Actions are essentially to create a complex tree object for presenting the parser tree.

The query tree object is sent to query execution. Different nodes of the query tree object are sent to different query execution cornponents, including: return pattern extraction, result pattern matcher, query join graph execution and condition filter. How these components work together is shown in Figure 4.9. 


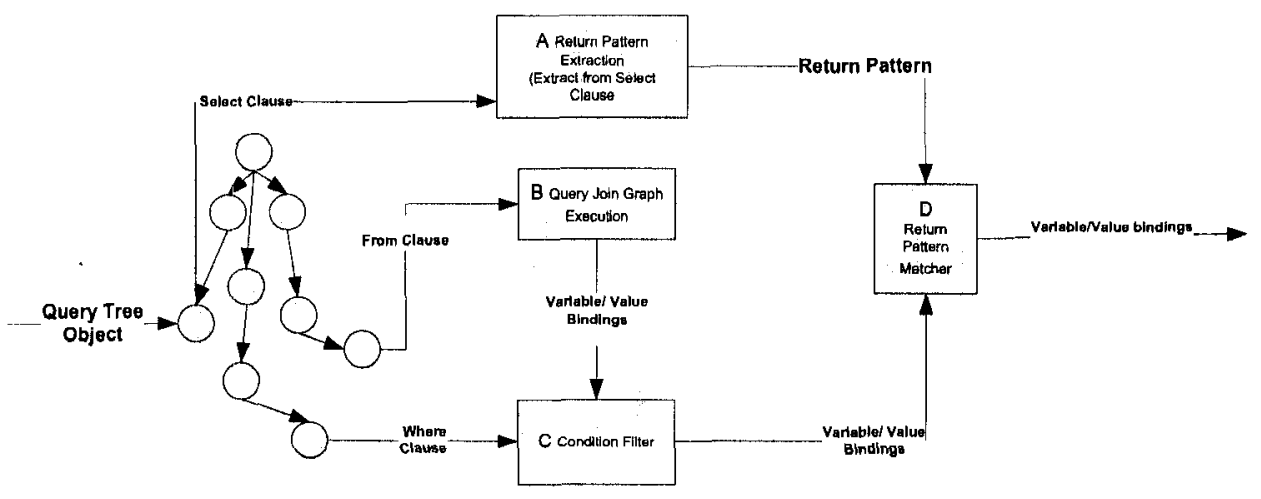

Figure 4.9: Query Execution

\subsubsection{Execution Components}

Return Pattern Execution It extracts variables from the select clause. The final result should be variable/value bindings, whose variables are listed in select clause.

Query Join Graph Execution It extracts the from clause of a structured query. The from clause is transferred to become a set of information item path expressions. We search for all information items that conform to the information path expression. For each information item, we get a set of variable/value bindings. We then join those variable/value bindings.

Consider the following information item pattern expression:

Folder, Cook \$x[(name, Cook Book), (subFolder, \$y)];

File, Food \$y[(name, dish), (size, \$z)]

For example, we conduct an information item pattern expression over example data in Figure 4.3. The execution processing is shown in Figure 4.10. Query join graph execution first extracts two information item path expressions. For each information path expression, 


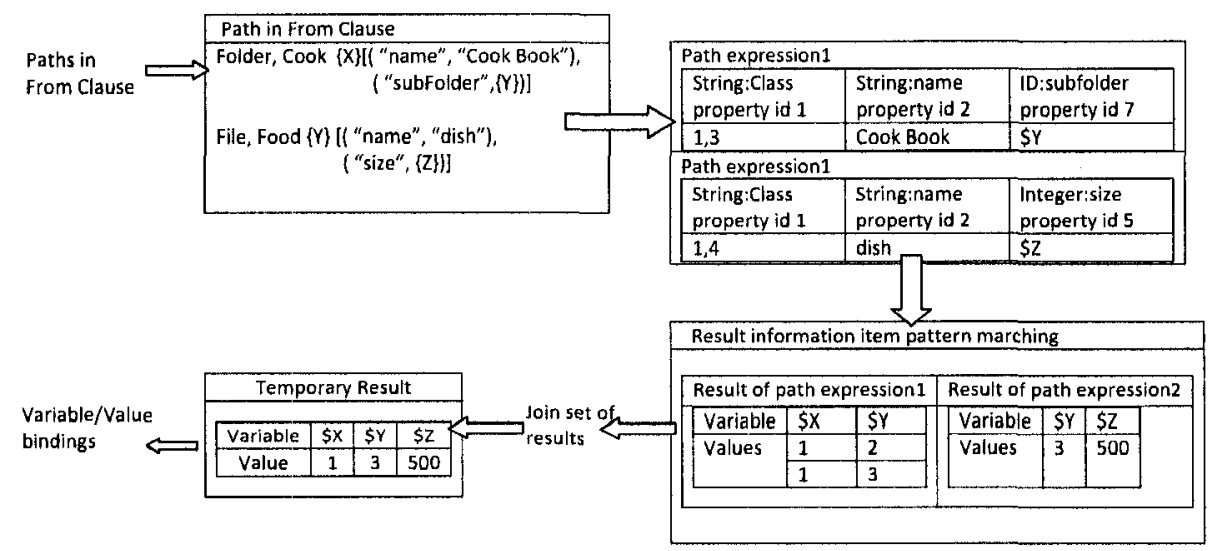

Figure 4.10: Query Join Graph Execution

we search for information items that match the path expression. For the first one, we search for the information items \$ $\mathrm{x}$ that belong to classes Folder and Cook, have the property name of value Cook Book and have the property subFolder in the entities database. The value of subFolder is $\$ y$. We find that the information item with the id 1 matches the path expression. Variable \$y is assigned with value 1. The information item called Cook Book has subfolders with id 2 and 3. Variable $\$ y$ is assigned with value 2 and 3. For the second information item path expression, we search for information items \$y which belong to classes File and Food, have the property name of value dish and have the property size in entities database. The value of size is $\$ z$. We find that information item with id 3 matches the path expression. Variable \$y is assigned with value 3. Information item called Dish has the size property with the value 500 . Variable $\$ z$ is assigned with value 500 . We join two results matched to those two information item path expressions and finally get variable/value binding $\{\{\$ \mathrm{x}, 1\},\{\$ \mathrm{y}, 3\},\{\$ \mathrm{z}, 500\}\}$. 
Condition Filter It gets the condition from the where clause, which eliminates the values that do not satisfy the condition.

For example, we require variable $\$ \mathrm{z}$ to be larger than 300 in the previous example. Condition filter will check if, in the result set, the value of variable $\$ \mathrm{z}$ is larger than 300 . If it is not larger than 300 , then the variable/value binding $\{\{\$ \mathrm{x}, 1\},\{\$ \mathrm{y}, 3\},\{\$ \mathrm{z}, 500\}\}$ will be removed from the result set.

Return Result Matcher It matches up result set outputted from the condition filter and transfer the result into the return pattern: Variable/Value bindings.

\subsubsection{Class Execution in Query Join Graph Execution}

The execution for matching classes for an information item is a special processing in query join graph execution, as the object of the property String:Class consists of class ids which is different from other objects of properties. Other properties' objects are values of those properties. We give sorne examples to explain how to process class information for an information item gradually. All examples are based on example shown in Figure 4.3 and the class information in Figure 4.3 is shown in Figure 4.11.

(1) Folder, Cook $\$ \times[]$

Result: $\$ \mathrm{x}=1$

(2) File, Recipe $\$ \times[]$

Result: $\$ \mathrm{X}=\{4,5\}$

(3) $\$ y \$ x[($ name, Cook Book)]

Result: $\$ \mathrm{x}=1 ; \$ \mathrm{y}=\{$ Folder, Cook $\}$

(4) $\$ y$ \$x[(name, Dish)]

Result: $\$ \mathrm{x}=3 ; \$ \mathrm{y}=\{$ Folder, Cook, Food $\}$

In the first example, we ask for the information item $\$ \mathrm{x}$ which belongs to classes Folder 


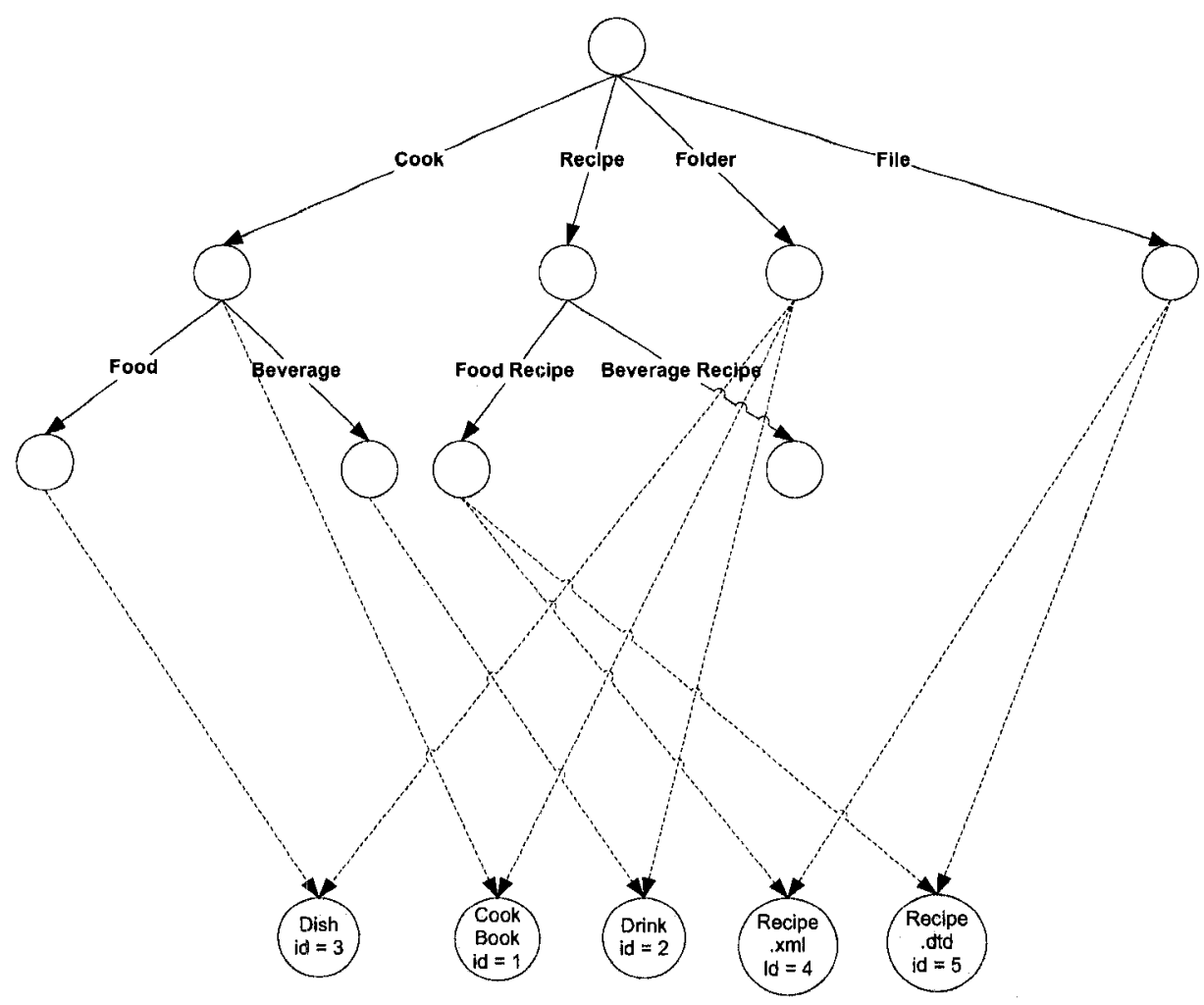

Figure 4.11: Classes Information 
and Cook. We fist look for the property id of the property String:Class in the property database and the id is 1 . We then look for class id of Folder and Cook in class database. The id of class Folder is 1 and the id of class Cook is 3. With property id 1, class ids 1 and 3 , we look for information matching information items in entity database. We finally find that information item with id 1 matches.

The third example asks for the information item $\$ \mathrm{x}$ which belongs to classes File and Recipe. As class Recipe has descendant classes Food Recipe and Beverage Recipe, the information item pattern expression is divided into two information item pattern expressions:

File, Food Recipe \$x[]; and

File, Beverage Recipe $\$ x[]$.

For the first expression, information items called Recipe.xml and Recipe.dtd match and for the second expression, there is no matching result. Hence, the result is a set $\{4,5\}$. Note that, all of classes and their descendants should be considered.

The third example asks for class names \$y for information item \$x called Cook Book. We first look for property id of property String:Class and its id is 1 ; we look for property id of property String:name and its id is 2 . We look for information items which have property with value id 2 and 1, and the object of the property with id 1 is Cook Book. We find that information item with id 1 matches and ids of its classes are 1 and 3. We than look for classes with id 1 and 3 in class database and the results of variable \$y are Folder and Cook.

The fourth example asks for class names $\$ y$ for information item $\$ \mathrm{x}$ called dish. Its processing is similar to the second example but there is a core difference, which is that after we get result Folder and Food, the processing here is not done yet. We also need to check in subclass database to see if class Folder and Food have parent classes. We find that class Food has parent class Cook. So, the results of \$y should be Folder, Food and Cook. Note that, all of classes and their ancestors should be considered.

Two core algorithms in class execution are shown in Figure 4.12 and Figure 4.13. The first algorithm gets ids of the class and its descendant classes; the second algorithm gets ids 


\begin{tabular}{|c|c|}
\hline \multicolumn{2}{|c|}{$\begin{array}{l}\text { Algorithm: getDescendantClassIDs } \\
\text { Input: classID } \\
\text { Output: classIDs (Vector<Integer }>\text { ) }\end{array}$} \\
\hline 1 & Initialize classIDs to an empty Vector<Integer $>$ \\
\hline 2 & Add classID to classIDs \\
\hline 3 & get ids of subclass for classID in subclass database to subclasses \\
\hline 4 & IF subclasses is not null THEN \\
\hline 4 & FOR each subclass of subclasses \\
\hline 6 & Call getDescendantClassIDs with value subclass returning classIDs1 \\
\hline 7 & IF classIDs1 is not empty THEN \\
\hline 8 & Add classIDs1 to classIDs \\
\hline 9 & ENDIF \\
\hline 10 & ENDFOR \\
\hline 11 & ENDIF \\
\hline 12 & Return classIDs \\
\hline
\end{tabular}

Figure 4.12: Algorithm of getting descent classes

of the class and its ancestor classes.

\subsubsection{Keyword Search}

Keyword search is a user-friendly search method. Users might only remember a word of a document that they want. In this case, users will input this word to the search engine. It will then search the word in the index, which might store the word as a key. If the search engine finds the word, it will further extract information items which have the word in the title, subject, content and so on. The disadvantage of this kind of keyword search is that it does not support accurate query results. The query result of the keyword search is usually too large and users must manually conduct the second query, to filter the query result. In order to provide a user-friendly and a more accurate query, we modified this kind of keyword search. 


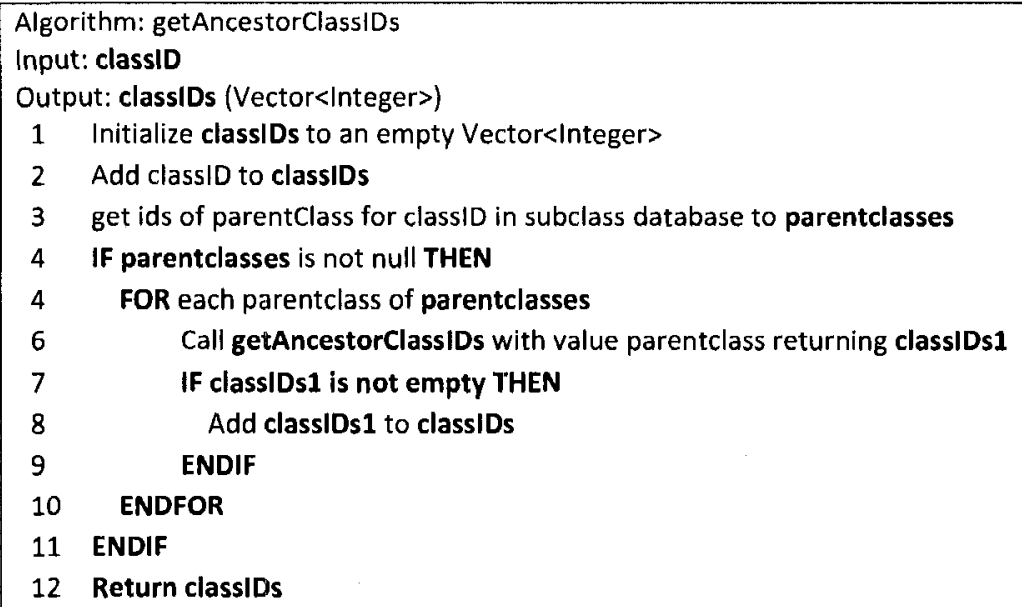

Figure 4.13: Algorithm of getting ancestor classes

Due to the fact that indexing content of a document has been already a sophistic technique, what we emphasize here is not to index the content of the information item while we attempt to index the meta data of a Folder information item, which includes a file name, path, and a relationship with other information items.

The main idea of building an index for meta data is not only to index the values of the meta data, but also to index the meta data's name and its values together. The index schema is shown in Table 4.1. Based on the example listed in Figure 4.3, we provide an illustration of the inverted index in Table 4.2. In the example, the keyword is an object or a string consisting of the property and its object. The value of the keyword is a set of ids of information items that have the keyword in its object or have the keyword as its property and object.

Note that meta data value for meta data String:Class consists of ids of those classes and all classes; its ancestors and descendants are indexed as well. In example Figure 4.3, 


\begin{tabular}{|l|l|}
\hline Keyword(object) & ID1,ID2 ..... \\
\hline /meta name(property)/Keyword(object) & ID3,ID4...... \\
\hline
\end{tabular}

Table 4.1: keyword inverted index

information items which belong to class Cook include: Cook Book, Dish and Drink. Class Cook is the ancestor class of class Food, to which information item Dish belongs. Class Cook is also the ancestor class of class Beverage, to which information item Dish belongs. Therefore, value of key /1/cook comprises ids of Cook Book, Dish and Drink, which are id 1, 2 and 3. Information items which belong to class Food include: Dish. Information items which belong to class Food Recipe, which contains string "food", include: Recipe.xml and Recipe.dtd. Therefore, value of key /1/food comprises ids of Dish, Recipe.xml and Recipe.dtd, which are id 3,4 and 5.

\subsubsection{Keyword Search Processing}

The input of the keyword search comprises of a set of (property, object) units and a set of terms. The input form has the following declaration:

$$
\overbrace{\left(p_{1}, k_{1_{1}} \ldots k_{1_{m}}\right) ; \ldots ;\left(p_{n}, k_{n_{1}} \ldots k_{n_{m}}\right)} ; \overbrace{t_{1} ; \ldots ; t_{l}}
$$

where $p_{i}$ is a value that is to be matched with a property name for $1 \leq i \leq n ; k_{i_{j}}$ is a value that is to be matched with the object of $p_{i}$ for $1 \leq i \leq n$ and $1 \leq j \leq m$; $t_{y}$ is a keyword that is to be matched in content for $1 \leq y \leq l$. Note that it is not required that $\overbrace{\left(p_{1}, k_{1_{1}} \ldots k_{1_{m}}\right) ; \ldots ;\left(p_{n}, k_{n_{1}} \ldots k_{n_{m}}\right)}$ and $\overbrace{t_{1} ; \ldots ; t_{l}}$ exist at the same time but it is required that one of them has to exist. 


\begin{tabular}{|c|c|}
\hline cook & 1 \\
\hline$/ 2 /$ cook & 1 \\
\hline book & 1 \\
\hline /2/book & 1 \\
\hline drink & 2 \\
\hline /2/drink & 2 \\
\hline dish & 3 \\
\hline /2/dish & 3 \\
\hline recipe & 4,5 \\
\hline /2/recipe & 4,5 \\
\hline $\mathrm{xml}$ & 4 \\
\hline$/ 2 / \mathrm{xml}$ & 4 \\
\hline$/ 8 / \mathrm{xml}$ & 4 \\
\hline dtd & 5 \\
\hline$/ 2 / d t d$ & 5 \\
\hline$/ 8 / \mathrm{dtd}$ & 5 \\
\hline folder & $1,2,3$ \\
\hline /1/folder & $1,2,3$ \\
\hline file & 4,5 \\
\hline$/ 1 /$ file & 4,5 \\
\hline /1/cook & $1,2,3$ \\
\hline$/ 1 /$ food & $3,4,5$ \\
\hline /1/beverage & 3 \\
\hline /1/recipe & 4,5 \\
\hline$\ldots$ & \\
\hline
\end{tabular}

Table 4.2: Example of the Inverted Index 
A keyword search that asks for the information item whose property name's value contains recipes and dish is as follows:

(name, recipes dish)

A keyword search asks for the information item whose property name's value contains string "recipe"; who has class containing strings "file" and has class containing string "food"; who contains the keyword "delicious" in its content. This keyword search's input is as follows:

(name, recipe); (class, file food); delicious

The keyword processing is shown in Figure 4.14. A user's input is decomposed into a set of (property, keywords) and keywords. Each (property, keywords) pair consists of a property and one or several keywords. A (property, keywords) pair is divided into a set of (property, keyword) pairs. Each (property, keyword) pair is transferred to be a string /property/keyword. The string, which is a term or a (property, keyword) pair is sent to the index searcher. The index searcher searches the inverted index storing keywords and their relative ids. If the index searcher finds the /property/keyword in the inverted index table, it returns ids of matched information items. After that, all ids returned by the index searcher are joined and the joined ids are then returned.

A keyword search that processes over example data stored in Table 4.2 is shown in Figure 4.15. We search for a file, whose name contains keyword "recipe" or "dtd". The result returned is the file recipe.dtd whose id is 5 .

\subsection{User interfaces}

We develop interfaces for users to manage their personal information. We present an example to illustrate how to use such interfaces. Specifically, we import example data by selecting path of the folder called test in which we put example data illustrated in Figure 3.11 . 


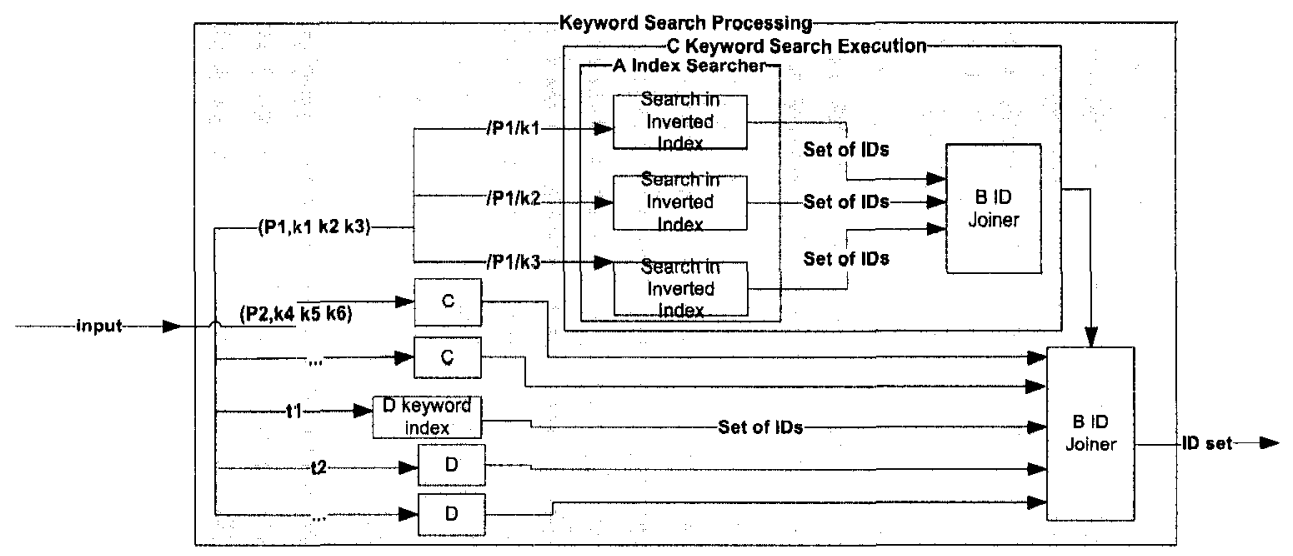

Figure 4.14: Keyword Search Processing

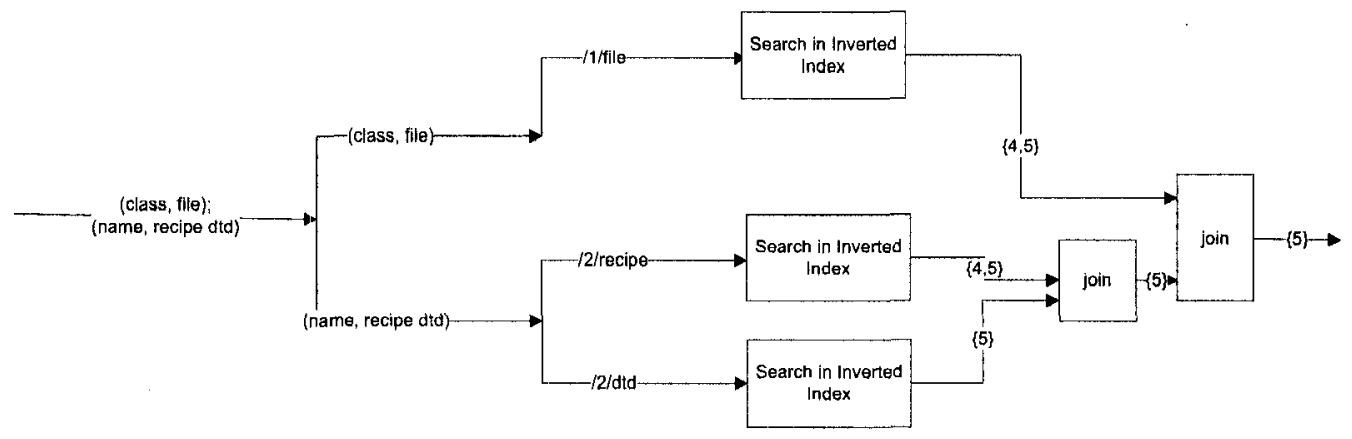

Figure 4.15: Keyword Search Processing Example 


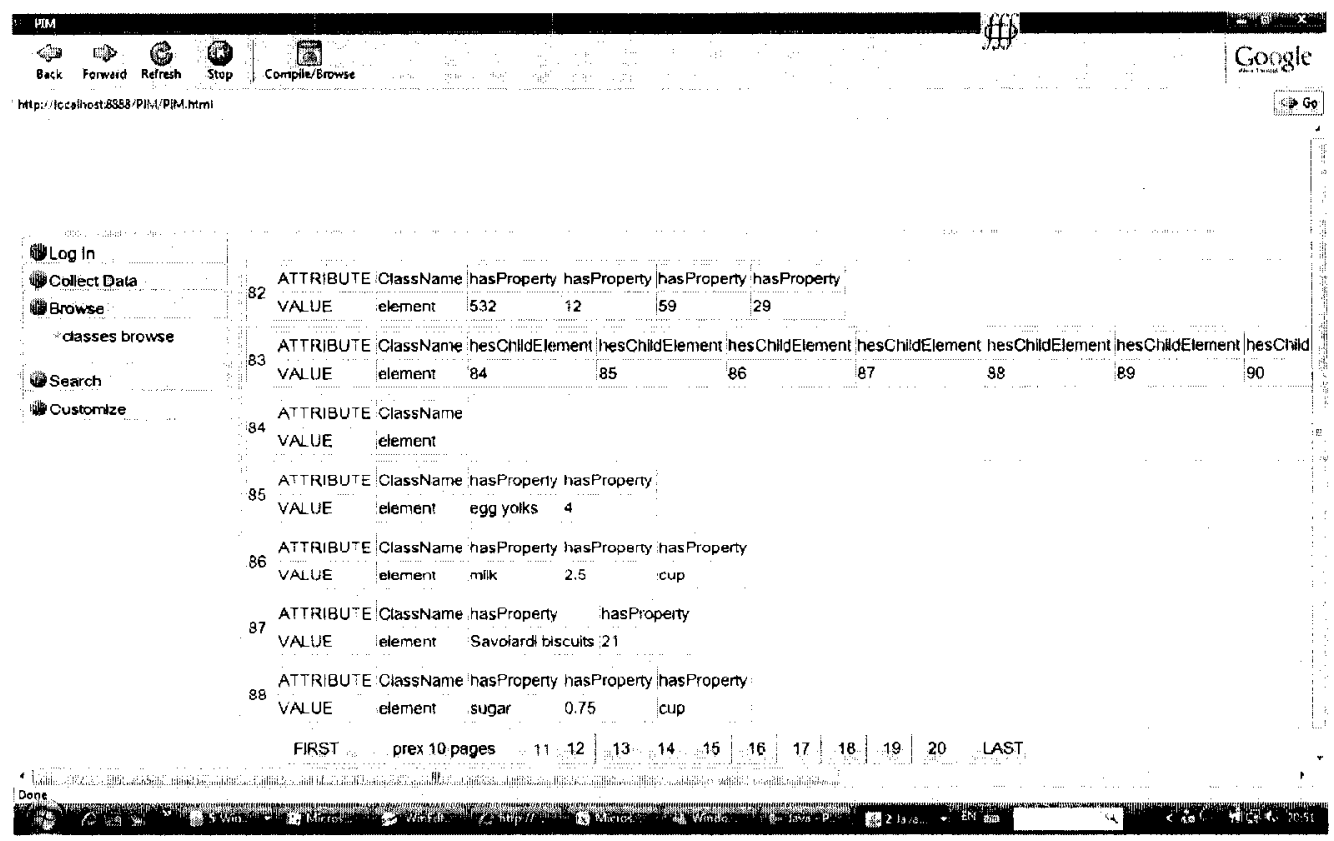

Figure 4.16: Browse console

We can also browse all of the information items in the browse console as shown in Figure 4.16.

We classify all file information items according to their different document types in default. There are three files in example data: dtd, XML and pdf. We can browse files within these three types as in the classification console, illustrated in Figure 4.17.

We can conduct keyword searches in keyword search console. For example, if we text "cook" then we get the result shown in Figure 4.18. The information item cook book contains files and folders. The keyword search console also shows files and folders contained 


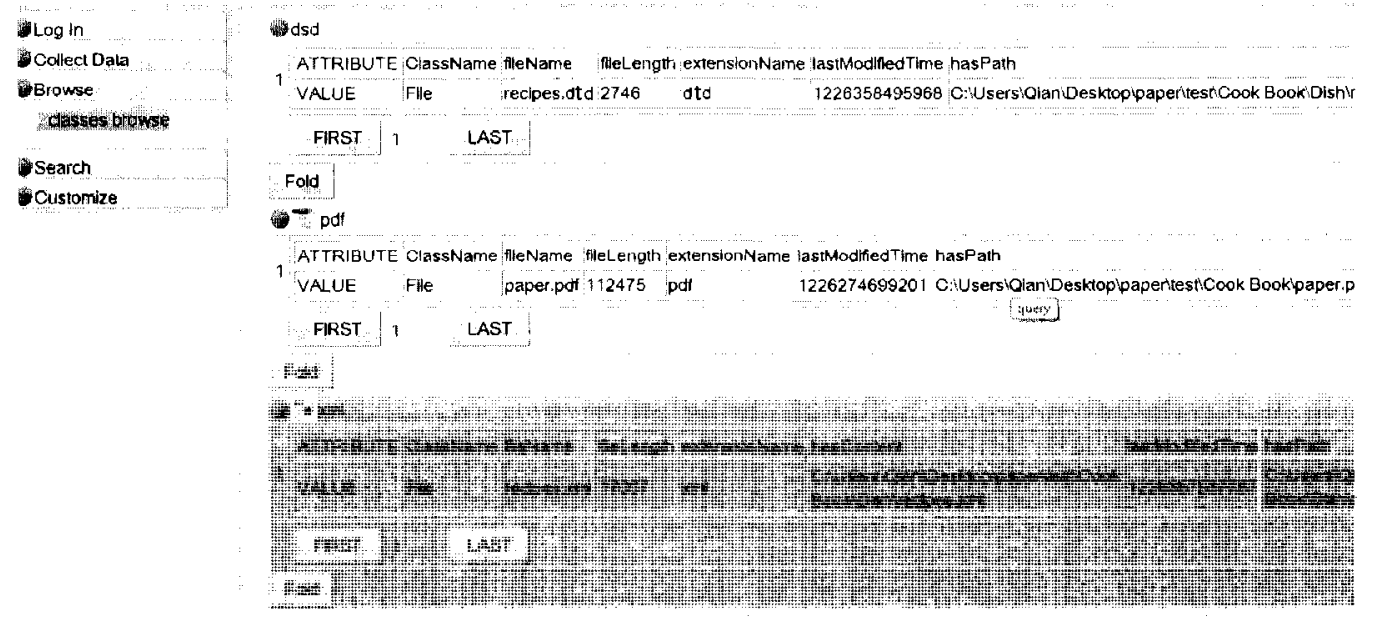

Figure 4.17: Classification console 

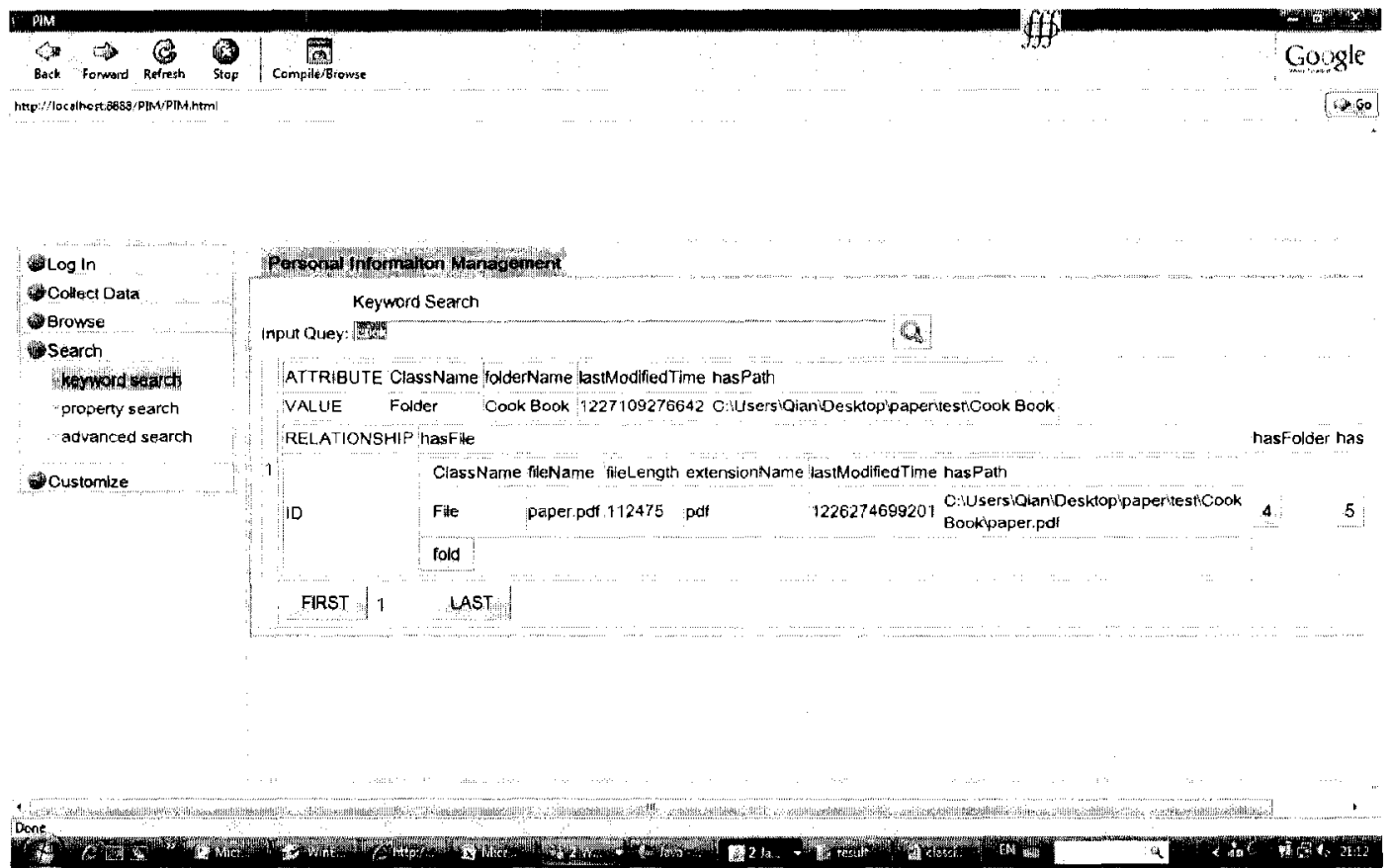

Figure 4.18: Keyword search console

in folder cook book.

We can conduct a property search in the property search console, which provides a manu that allows users to select the property inside. Users can then input the value of the properties to search for information items. For example, we select property String:extensionName and input "xml", and we get the result illustrated in Figure 4.19.

We can conduct a structured query in the advanced search console. For example, we can input the following query to get the names of all the folders:

$$
\begin{array}{ll}
\text { select } \$ y \\
\text { from } & \text { Folder } \$ x[(\text { name, } \$ y)]
\end{array}
$$




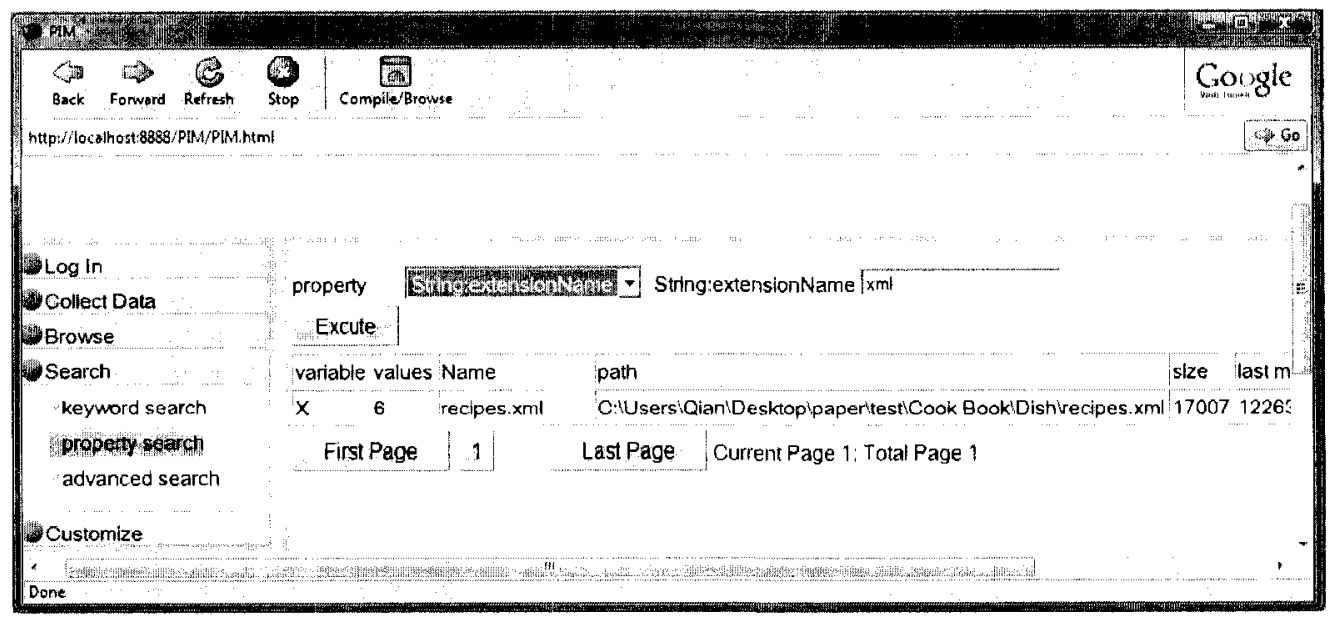

Figure 4.19: Property search console

Figure 4.20 demonstrates that we can attain four folders test, cook book, Dish and Drink.

\subsection{Experimental results}

Our experimental platform is a Windows Vista operation system that runs on a $2.0 \mathrm{GHz}$ Intel Pentium PC with $2 \mathrm{G}$ of physical memory. We import personal information from a personal computer. Personal information includes data stored in XML documents, emails from email client outlook, data stored in Mysql database and data in file systems.

Table 4.3 records the extraction time, the number of information items and the database size. Figure 4.21 illustrates the extraction time and the size of the database scale as the number of information items scales.

We conduct the following query which asks for names of all files stored in storages 


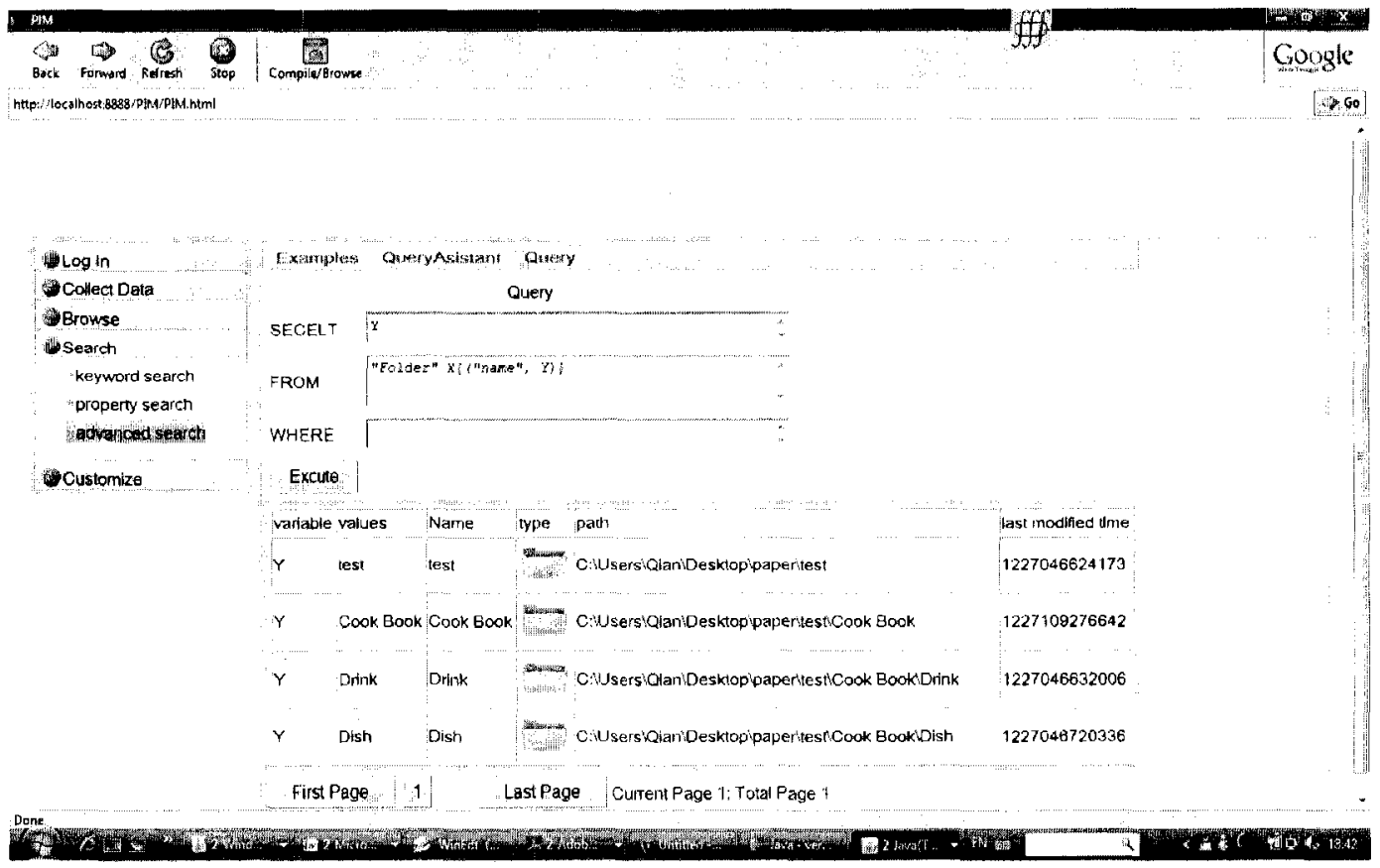

Figure 4.20: Structured query console

\begin{tabular}{|c|c|c|}
\hline Extraction time(Milli second) & Number of information items & Size of database(MB) \\
\hline 2419 & 903 & 1.52 \\
\hline 6293 & 3613 & 10.3 \\
\hline 56955 & 32,483 & 23 \\
\hline 443150 & 145,421 & 114 \\
\hline
\end{tabular}

Table 4.3: Extraction time and size of database as number of information items scales 

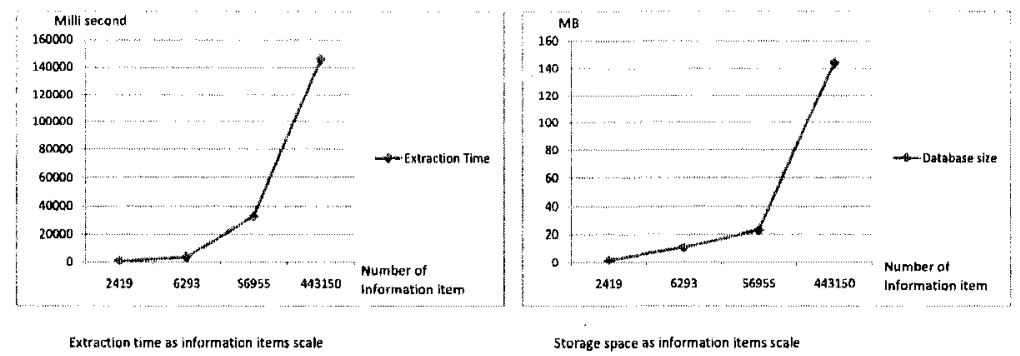

Figure 4.21: Extraction time and size of database as number of information items scales

with different sizes. We record query times and illustrate how the query time changes as the number of information items scales in Figure 4.22 . We can observe that the query speed slows down as the number of information items scales.

select \$y

from File $\$ x[($ name, $\$ y)]$ 


\begin{tabular}{|c|c|c|}
\hline Number of information item & Number of returned values & Query time (milli second) \\
\hline 903 & 840 & 79 \\
\hline 3613 & 3357 & 199 \\
\hline 32,483 & 28,095 & 4537 \\
\hline 145,421 & 125,369 & 15470 \\
\hline
\end{tabular}

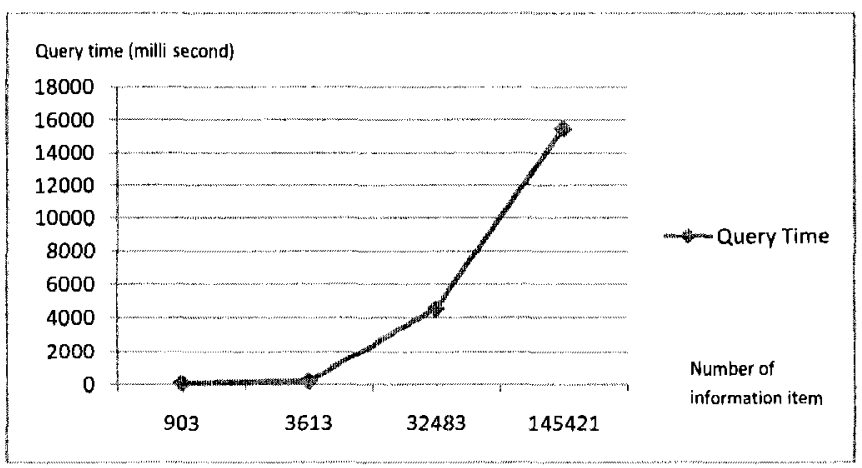

Figure 4.22: Query time as number of information items scales 


\section{Chapter 5}

\section{Conclusion}

In order to organize heterogeneous data sources in an effective and easy way, we have proposed a PIM Model in this thesis. It has the following features:

- Data in heterogeneous data sources have different formats and diverse data types and it is difficult to represent them in a single table. Our PIM Model makes representation of such data easy by reducing the effort of predefining the schema. Heterogeneous data are converted to information items. Through their ids, information items can be connected to form a uniform view.

- Information items can belong to multiple classes and classes can be organized hierarchically.

- Dataspace requires representation of heterogeneous data in a uniform way, requires data to be integrated loosely, requires to provide services upon coexistent data and allows coexistent data to be integrated further. Our PIM Model represents heterogeneous data in a uniform graph view. Over the view, structured query and keyword search services are able to be provided. The view has simple format and it allows semantic integration upon it. Therefore, it fulfills the Dataspace vision. 
Based on the PIM Model, we have designed and implemented a Personal Information Management system, which has following features:

- It has wrappers to extract data from various data sources such as file system, relational databases, XML documents and email servers or email clients. Though data residing at those data sources have different formats, our system converts them in a uniform format; that is, information item.

- It stores information items in Berkeley DB and supports indexes on primary database to speed up operations on the storage.

- It provides a structured query which is similar to SQL and supports searching information items in a uniform view. It also provides a keyword search which indexes properties and property values together in an inverted index table.

- It provides interfaces for users to collect, browse, search, classify personal information.

- It is platform-independent and extendable. We can add extractors when we need to extract data from other different data sources.

We can extend our system in the following ways:

- Develop programs to map our structured query language to other query languages so that we can search data stored in various applications without extracting data and storing it in our database.

- Optimize query execution for structured query and the keyword search. Apply more advanced query strategies which are more user friendly and return more accurate query results.

- Add data updating component to keep data stored in storage fresh. 
- Conduct semantic data integration over coexistent data to provide more advanced services.

Part of this research work has been published in [6]. 


\section{Bibliography}

[1] J. L. Beckmann, A. Halverson, R. Krishnamurthy, and J. F. Naughton. Extending rdbmss to support sparse datasets using an interpreted attribute storage format. In Proceedings of ICDE, page 58, 2006.

[2] S. Chawathe, H. Garcia_Molina, J. Hammer, K. Ireland, Y. Papakonstantinou, Jeffrey Ullman and J. Widom. The TSIMMIS Project: Integration of Heterogeneous Information Sources. In Proceedings of IPSJ Conference, page 7-18, Tokyo, Japan, October 1994.

[3] A. Y. Halevy. Answering Queries using views: A survey. In Very Large Database J,, page $270-294,2001$.

[4] R. Hull. Managing semantic heterogeneity in databases: A theoretical perspective. In Principle of Database Systems, 1997. In Proc. Of the 16th ACM SIGACT SIGMOD SIGART Symp, page 27-29, 1997.

[5] J. D. Ullman. Information integration using Logical views. In Proc, of the 6th Int. Conf. on Databses Theory, In volume 1186 of Lecture Notes in Computer Science, pages 19-40, Springer, 1997.

[6] M. Zhong, M. Liu and Q. Chen. Modeling Heterogeneous Data in Dataspace. In IEEE IRI-2008, 2008 IEEE International Conference on Information Reuse and Integration, p 404-409, 2008. 
[7] xml.apache.org. Apache Xindice.

[8] HP Labs Semantic Web Research. A Semantic Web Framework for Java.

[9] mysql.com. MySql. http://www.mysql.com/.

[10] oracle.com. Oracle Database. http://www.oracle.com/.

[11] Code.Google.com Google Web Toolkit. http://code.google.com/.

[12] Oracle.com Berkeley DB. http://www.oracle.com/technology/products/berkeley-db/.

[13] Google, Inc. Google Desktop. http://desktop.google.com/.

[14] Yahoo, Inc. Yahoo! Desktop. http://info.yahoo.com/privacy/in/yahoo/desktopsearch/.

[15] Copernic, Inc. Copernic Desktop. http://www.copernic.com/.

[16] Microsoft.org. Window Desktop Search.

http://www.microsoft.com/windows/products/winfamily/desktopsearch/.

[17] G. Karvounarakis, S. Alexaki, V. Christophides, D. Plexousakis, and M. Schol. RQL: A Declarative Query Language for RDF. In Proceedings of the Eleventh International World Wide Web Conference (WWW'02), Honolulu, Hawaii, USA, May7-11 2002.

[18] Jeen Broekstra and Arjohn Kampman. SeRQL: An RDF Query and Transformation Language. In International Semantic Web Conference, ISWC 2004, 2004.

[19] Andy Seaborne. Rdql - a query language for rdf. In w3c, 2004. http://www.w3.org/Submission/2004/SUBM-RDQL-20040109/.

[20] M. Sintek and S. Decker. TRIPLE - an RDF query, inference and transformation language. In Deductive Databases and Knowledge Management (DDLP), 2001. 
[21] M. Sintek and S. Decker. TRIPLE - an RDF query, inference and transformation language. In Deductive Databases and Knowledge Management (DDLP), 2001.

[22] J. Broekstra, A. Kampman, and F. van Harmelen. Sesame: A Generic Architecture for Storing and Querying RDF and RDF Schema. In ISWC, pages 54C68, 2002.

[23] M. Kifer, G. Lausen, and J. Wu. Logical foundations of object-oriented and framebased languages. In Journal of the ACM, 42, 1995.

[24] P. Haase, J. Broekstra, A. Eberhart, R. Volz. A comparison of RDF Query Lanuages. In $I S W C^{\prime} 04$, Germany, 2004.

[25] Haystack: A Customizable General-Purpose Information Management Tool for End Users of Semistructured Data. In CIDR, 2005.

[26] http://msdn.microsoft.com/data/WinFS. WinFS.

[27] Yuhan Cai, Xin Dong, Alon Y. Halevy, Jing Liu and Jayant Madhavan. Personal Information Management with SEMEX. In SIGMOD DEMO, 2005.

[28] D. Huynh, D. Karger, D. Quan Haystack: A Platform for Creating, Orgnizing and Visualizing Information Using RDF. In Semantic Workshop, Hawaii, USA, 2002.

[29] D. J. Abadi, A. Marcus, S. R. Madden, and K. Hollenbach. Scalable semantic web data management using vertical partitioning. In Proceedings of $V L D B$, pages 411 422, 2007.

[30] G. P. Copeland and S. N. Khoshafian. A decomposition storage model. In Processings of SIGMOD, pages 268-279, 1985.

[31] M. Lansdale. The psychology of personal information management. In Applied Ergonomics, 19(1):55-66, 1988. 
[32] V. Bush. As we may think, July, 1945.

[33] L. Blunschi, J. Dittrich, O. Girard, S. K. Karakashian and M. A. V. Salles. A Dataspace Odyssey: The iMeMex Personal Dataspace Management System, Asilomar, California, USA, 2007.

[34] J. Gemmell, G. Bell, and R. Lueder. Mylifebits: A personal database for everything. Com- munications of the ACM, 49(1):88-95, 2006.

[35] S. Al-Khalifa, H. Jagadish, N. Koudas, J. M. Patel, D. Srivastava, and Y. Wu. Structural joins: A primitive for efficient XML query pattern matching. In ICDE, 2002.

[36] N. Bruno, N. Koudas, and D. Srivastava. Holistic twig joins: Optimal XML pattern matching. In Sigmod, 2002.

[37] R. Baeza-Yates and G. Gonnet. Fast text searching for regular expressions or automaton simulation over tires. In Journal of the ACM, 43(6):915C936, 1996.

[38] Z. Chen, J. Gehrke, F. Korn, N. Koudas, J. Shanmugasundaram, and D. Srivastava. Index structures for matching xml twigs using relational query processors. In ICDE Workshops, 2005.

[39] R. Goldman and J. Widom. Dataguides: Enabling query formulation and optimization in semistructured databases. In Proc. of $V L D B$, Athens, Greece, 1997.

[40] T. Milo and D. Suciu. Index structures for path expressions. In Proc. of ICDT, 1999.

[41] H. Wang, S. Park, W. Fan, and P. S. Yu. ViST: A dynamic index method for querying XML data by tree structures. In Proc. of SIGMOD, 2003.

[42] V. Hristidis and Y. Papakonstantinou. DISCOVER: Keyword search in relational databases. In $V L D B, 2002$. 
[43] J. Graupmann, R. Schenkel, and G. Weikum. The SphereSearch engine for unified ranked retrieval of heterogeneous XML and web documents. In $V L D B, 2005$.

[44] M. Sayyadian, H. Lekhac, A. Doan, and L. Gravano. Efficient keyword search across heterogeneous relational databases. In ICDE, 2007.

[45] X. Dong, A. Halevy Indexing Dataspaces. In SIGMOD07, June 11-14, Beijing, 2007.

[46] Y. Li , X. Meng. Research on Personal Dataspace Management. In Proceedings of the Second SIGMOD PhD Workshop on Innovative Database Research (IDAR 2008), June 13, 2008, Vancouver, Canada.

[47] J. P. Dittrich and M. A. V. Salles. iDM: A Unified and Versatile Data Model for Personal Dataspace Management. In $V L D B, 2006$.

[48] M. Banko, M. J. Cafarella, S. Soderland, M. Broadhead, and O. Etzioni. Open information extraction from the web. In Proceedings of IJCAI, 2007.

[49] O. Etzioni, M. Cafarella, D. Downey, A.-M. Popescu, T. Shaked, S. Soderland, D. S.Weld, and A. Yates. Unsupervised named-entity extraction from the web: an experimental study. In Artificial Intelligence, 165(1):91-134, June 2005.

[50] W. Jones, H. Bruce. A Report on the NSF-Sponsored Workshop on Personal Information Management, Seattle, WA, 2005.

[51] Microsoft Corporation. Windows Future Storage. http://msdn.microsoft.com/enus/library/aa480687.aspx. 